\title{
Hi observations of the nearest starburst galaxy NGC 253 with the SKA precursor KAT-7
} \author{
T. A. Oosterloo ${ }^{2,3}$ and G. H. Heald ${ }^{2,3}$ \\ ${ }^{1}$ Department of Astronomy, University of Cape Town, Private Bag X3, Rondebosch 7701, South Africa \\ ${ }^{2}$ Netherlands Institute for Radio Astronomy (ASTRON), Postbus 2, 7990 AA Dwingeloo, The Netherlands \\ ${ }^{3}$ Kapteyn Astronomical Institute, University of Groningen, PO Box 800, 9700 AV Groningen, The Netherlands
}

D. M. Lucero ${ }^{1 \star}$, C. Carignan ${ }^{1}$, E. C. Elson ${ }^{1}$, T. H. Randriamampandry ${ }^{1}$, T. H. Jarrett ${ }^{1}$,

\begin{abstract}
We present HI observations of the Sculptor Group starburst spiral galaxy NGC 253, obtained with the Karoo Array Telescope (KAT-7). KAT-7 is a pathfinder for the SKA precursor MeerKAT, under construction. The short baselines and low system temperature of the telescope make it very sensitive to large scale, low surface brightness emission. The KAT-7 observations detected 33\% more flux than previous VLA observations, mainly in the outer parts and in the halo for a total $\mathrm{HI}$ mass of $2.1 \pm 0.1 \times 10^{9} \mathrm{M}_{\odot}$. HI can be found at large distances perpendicular to the plane out to projected distances of $\sim 9-10 \mathrm{kpc}$ away from the nucleus and $\sim 13-14 \mathrm{kpc}$ at the edge of the disk. A novel technique, based on interactive profile fitting, was used to separate the main disk gas from the anomalous (halo) gas. The rotation curve (RC) derived for the HI disk confirms that it is declining in the outer parts, as seen in previous optical Fabry-Perot measurements. As for the anomalous component, its RC has a very shallow gradient in the inner parts and turns over at the same radius as the disk, kinematically lagging by $\sim 100 \mathrm{~km} / \mathrm{sec}$. The kinematics of the observed extra planar gas is compatible with an outflow due to the central starburst and galactic fountains in the outer parts. However, the gas kinematics shows no evidence for inflow. Analysis of the near-IR WISE data, shows clearly that the star formation rate (SFR) is compatible with the starburst nature of NGC 253.
\end{abstract}

Key words: techniques: interferometric - galaxies: individual: NGC 253 - galaxies: starburst - galaxies: kinematics and dynamics - galaxies: haloes

\section{INTRODUCTION}

The seven-dish KAT-7 array (Carignan et al. 2013) was built as an engineering testbed for the 64-dish Karoo Array Telescope, known as MeerKAT, which is the South African precursor of the Square Kilometre Array (SKA). KAT-7 and MeerKAT are located close to the South African SKA core site in the Northern Cape's Karoo desert region. Construction of the array was completed in December 2010 . The array is extremely compact, with baselines ranging from $26 \mathrm{~m}$ to $185 \mathrm{~m}$ and the receivers have a very low $T_{\text {sys }} \sim 30 \mathrm{~K}$. While its main purpose is to test technical solutions for MeerKAT and the SKA, scientific targets, such as NGC 253, were also observed during commissioning to test the HI line mode. In this paper, we present over 150 hours of observations taken with KAT-7 in order to study its large scale extra-planar HI gas component.

NGC 253 is the nearest example of a galaxy undergoing enhanced star formation in the South, M82 being the nearest one in

\footnotetext{
*dlucero@ast.uct.ac.za
}

the North. Its nucleus contains a starburst with a star-formation rate (SFR) of $\sim 5 \mathrm{M}_{\odot} \mathrm{yr}^{-1}$, roughly $70 \%$ of the rate of the entire galaxy (Wik et al. 2014). It is considered a prototype starburst galaxy (see e.g. Sakamoto et al. 2011; Strickland et al. 2002) and Rieke, Lebofsky \& Walker (1988) suggest that it may be in an earlier starburst phase than M82. Of particular interest is that, despite the high SFR, NGC 253 is a non-interacting (non-merger) system, which does not exclude that it occurred in the past (see Sec. 7.4).

One very interesting feature is the diffuse extra-planar HI surrounding the X-ray emission, first seen in the ATCA observations of Boomsma et al. (2005). Not only is the nuclear starburst forming stars at a fairly high rate but it is also thought to produce a super-wind (Heckman, Armus \& Miley 1990), which expels material into the halo. The high sensitivity of KAT-7 to large scale diffuse emission (Carignan et al. 2013) allows us to better study the known extra-planar gas component. Such extra-planar gas is expected when looking at a deep UV image showing emission far from the plane, compared to a 2MASS image (Jarrett et al. 2003), which shows the main stellar disk component (Figure 1). 


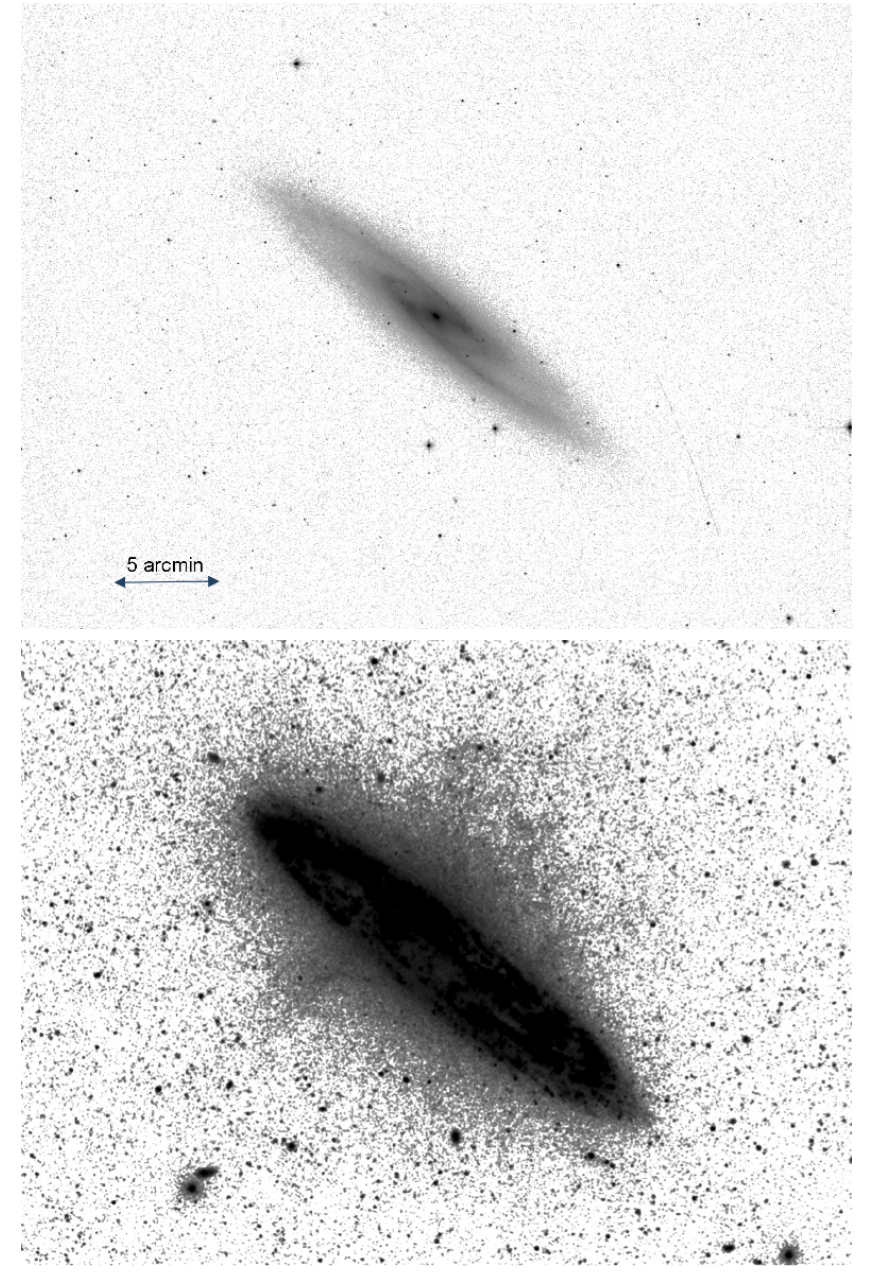

Figure 1. 2MASS K-band (top) and GALEX FUV (bottom) images of NGC 253.

In NGC 253 , most of the activity is confined within $\sim 1 \mathrm{kpc}$ of the nucleus, fuelled by bar-driven dense cold molecular gas that obscures the starburst (Rieke et al. 1980; Hougton et al. 1997; Engelbracht et al. 1998; Sakamoto et al. 2011). Recent X-ray, optical and ALMA mm-wave observations trace outflowing gas that is powered by the starburst (Mitsuishi, Yamasaki \& Takei 2013; Bolatto et al. 2013). Owing to proximity, the two nearest starbursts, NGC 253 and M82, are ideal laboratories for detailed (pc-scale) study of nuclear outflows and galactic-disk fountains. Conversely, their global properties - which place the starbursts in context with the total gas and stellar content - are a challenge to measure due to their large angular size and brightness (frequently saturating detectors). While large angular scales are not a problem at optical wavelengths, for radio interferometry imaging studies, one needs the proper short baselines to be able to detect large structures and the compactness of KAT-7 makes it better suited than e.g. the VLA for the observation of those large scales in nearby objects (see Table 2).

Recent deep HI observations of several nearby galaxies indicate that up to $15 \%$ of the neutral hydrogen of a spiral galaxy is located in the halo (Heald et al. 2014). The gas outside the main HI disk of spirals has been studied in many galaxies: NGC 891 (Swaters, Sancisi \& van der Hulst 1997; Oosterloo, Fraternali \& Sancisi 2007), NGC 2403 (Schaap, Sancisi \& Swaters 2000; Fraternali et al. 2001), NGC 2997 (Hess et al. 2009), NGC 3198 (Gentile et al. 2013), NGC 4244 (Zschaechner et al. 2011), NGC 4559 (Barbi- eri et al. 2005), NGC 4565 (Zschaechner et al. 2012), NGC 5775 (Lee et al. 2001), NGC 6946 (Boomsma et al. 2008), M31 \& M33 (Westmeier, Braun \& Thilker 2005; Thilker et al. 2004) and the Milky Way (MW) through High Velocity Clouds (HVCs) (Wakker \& van Woerden 1997), the Magellanic Stream (Mathewson, Cleary \& Murray 1974) and the Leading Arm (Putman et al. 1998). See also Heald et al. $(2011,2014)$ for a description of the HALOGAS survey and Sancisi et al. (2008); Putman, Peek \& Joung (2012) for reviews.

The interaction between the gas in the halo and the disk of spirals is believed to play an important role in their evolution. Halo gas connects the baryon-rich intergalactic medium (IGM) to the starforming disks of galaxies (Putman, Peek \& Joung 2012). The classical "galactic fountain" scenario (Schapiro \& Field 1976), where hot gas is expelled from the disk through "galactic chimneys" created by multiple supernova explosions from clusters of young massive stars (Normandeau, Taylor \& Dewdney 1996) can explain the presence of hot gas in the halo. It is believed that, as the gas expands, it eventually converts back to $\mathrm{HI}$ as it cools through radiative losses; raining back down on the disk (Bregman 1980) and feeding subsequent star formation (see also the models for the MW: Marasco, Fraternali \& Binney 2012). A good example of such exchanges between the disk and the halo is the $\mathrm{H} \alpha$ kinematical study by Cecil et al. (2001) of one of the filament in the super wind outflow of NGC3079. Long ago, Oort (1966) also suggested an external origin for some of that gas in the form of primordial gas clouds left over from the formation of the galaxies. Finally, as suggested by the Magellanic Stream and the Leading Arm in the MW, some of that halo gas could come from ISM torn out of dwarf galaxies during close encounters with a large spiral.

The optical and IR parameters of NGC 253 are summarized in Table 1. It is the brightest and earliest type among the five late-type spirals composing the nearest group to us, the Sculptor group (Puche \& Carignan 1988). It is classified SAB(s)c by de Vaucouleurs et al. (1991) but, as can be seen in Figure 2, it is clearly a barred SB(r)c galaxy on IR images. The different recent distance estimates from the Planetary Nebulae Luminosity Function (PNLF) and the Tip of the Red Giant Branch (TRGB) distance indicators (Rekola et al. 2005; Mouchine et al. 2005; Dalcanton et al 2009; Jacobs et al. 2009; Hislop et al. 2011) give a distance of $3.5 \mathrm{Mpc}$, for a scale of $\simeq 1 \mathrm{kpc}$ per arcmin. While NGC 55 and NGC 300 are on the near side and NGC 7793 on the far side of the Sculptor group, NGC 253 is at the mean distance of the group with NGC 247 , which is only at a projected distance of $\sim 350 \mathrm{kpc}$ to the North.

The first detailed HI observations of this galaxy (Huchtmeier 1972; Combes, Gottesman \& Weliachew 1977) show clearly an asymmetric HI distribution. The presence of a strong continuum source in the central regions (Hummel, Smith \& van der Hulst 1984) suggests that any kinematical analysis in the very inner parts is to be undertaken with great care. From the low spatial resolution single dish early Hi observations, the rotation curve was believed to be declining at large radii (Huchtmeier 1975) but higher resolution VLA observations (Puche, Carignan \& van Gorkom 1991) show a rotation curve still rising at the last observed velocity point $\left(\sim 12^{\prime}\right)$. However, recent Fabry-Perot observations (Bland-Hawthorn, Freeman \& Quinn 1997; Hlavacek-Larrondo et al. 2011) detected $\mathrm{H} \alpha$ emission further out than previous $\mathrm{HI}$ observations. While the $\mathrm{H} \alpha$ kinematics agree with the $\mathrm{HI}$ for $\mathrm{R} \leq 12^{\prime}$, it suggests a declining rotation curve between $15^{\prime}$ and $19^{\prime}$. It will be interesting to see if our KAT-7 observations extend to those radii and confirm this decline.

Figure 3 shows previous higher spatial resolution $\left(68^{\prime \prime} \times 68^{\prime \prime}\right.$ vs $213^{\prime \prime} \times 188^{\prime \prime}$ ) VLA observations (Puche, Carignan \& van 


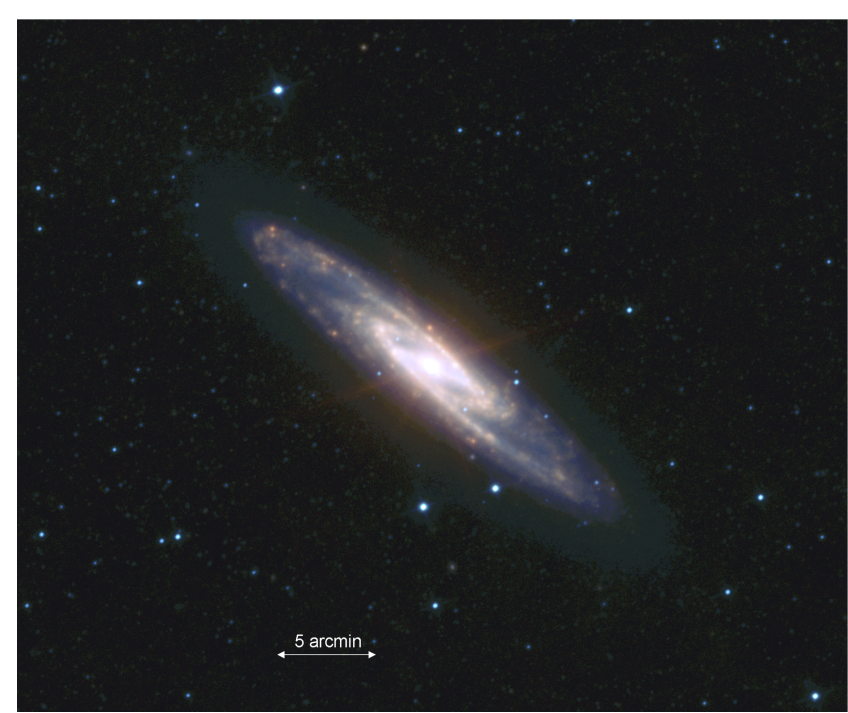

Figure 2. Wise W1 (blue), W2 (green), W3 (orange) and W4 (red) colorcombination image of NGC 253.

\begin{tabular}{|c|c|}
\hline Parameter & Value \\
\hline Morphological type $^{1}$ & $\mathrm{SB}(\mathrm{r}) \mathrm{c}$ \\
\hline Right Ascension ${ }^{2}$ (J2000) & $0^{\mathrm{h}} 47^{\mathrm{m}} 33.1^{\mathrm{s}}$ \\
\hline Declination $^{2}(\mathrm{~J} 2000)$ & $-25^{\circ} 17^{\prime} 18^{\prime \prime}$ \\
\hline Distance (Mpc) & $3.47 \pm 0.14$ \\
\hline Scale $\left(\mathrm{kpc} \operatorname{arcmin}^{-1}\right)$ & $\sim 1$ \\
\hline Isophotal major diameter ${ }^{2}, D_{25}$ & $27.5^{\prime}$ \\
\hline Holmberg radius ${ }^{3}, R_{H O}$ & $17.5^{\prime}$ \\
\hline Axis ratio $^{2}(\mathrm{q}=\mathrm{b} / \mathrm{a}), R_{25}$ & 0.25 \\
\hline Inclination $\left(\mathrm{q}_{0}=0.15\right)$ & $78.3^{0}$ \\
\hline Position Angle & $52^{0}$ \\
\hline Total apparent $B$ magnitude ${ }^{2}$ & 8.04 \\
\hline Corrected apparent $B$ magnitude ${ }^{2}$ & 7.02 \\
\hline Absolute $B$ magnitude & -20.68 \\
\hline Total B luminosity, $L_{\odot}$ & $2.78 \times 10^{10}$ \\
\hline Absolute $W 1$ magnitude, WISE & -24.24 \\
\hline Color (W1 - W2), WISE & 0.21 \\
\hline $\log \left(\mathrm{M}_{*}\right)$, WISE, $M_{\odot}$ & 10.33 \\
\hline
\end{tabular}

(1) See Figure 2

(2) de Vaucouleurs et al. (1991)

(3) Radius at $\mu_{B}=26.6 \mathrm{mag} \operatorname{arcsec}^{-2}$

Table 1: Optical \& IR (WISE) parameters of NGC 253.

Gorkom 1991). Note however that the final KAT-7 data have a factor of $\sim 4$ better velocity resolution. Regarding the HI distribution, the first feature to notice is the strong absorption in the centre, which prevents any reliable kinematics to be extracted close to the starburst region. It can be seen that besides tracing the spiral arms, the $\mathrm{HI}$ is really concentrated around the nucleus. As for the velocity field, it is a textbook case in terms of regularity. Only a slight increase in position angle is suggested in the very outer parts. The most interesting map is the velocity dispersion map, which shows a clear increase $\sim 45-50 \mathrm{~km} \mathrm{~s}^{-1}$ around the starburst region while it is fairly constant $\sim 10-15 \mathrm{~km} \mathrm{~s}^{-1}$ in the rest of the disk. Because of the short exposure time ( $\sim 30$ minutes in D configuration) and the lack of short spacings, those observations only show the disk emis-

\begin{tabular}{ccccc}
\hline \hline $\begin{array}{c}(1) \\
\text { Telescope }\end{array}$ & $\begin{array}{c}(2) \\
\text { Min. bl } \\
(\mathrm{m})\end{array}$ & $\begin{array}{c}\text { Ant. diam. } \\
(\mathrm{m})\end{array}$ & $\begin{array}{c}(4) \\
\text { LAS } \\
(\mathrm{min} . \mathrm{bl})\end{array}$ & $\begin{array}{c}(5) \\
\text { LAS } \\
\text { (sha. lim. })\end{array}$ \\
\hline KAT-7 & 26 & 12 & $16.7^{\prime}$ & $36.1^{\prime}$ \\
VLA-D & 35 & 25 & $12.4^{\prime}$ & $17.3^{\prime}$ \\
WSRT-MS & 36 & 25 & $12.0^{\prime}$ & $17.3^{\prime}$ \\
GMRT & 100 & 45 & $4.3^{\prime}$ & $9.6^{\prime}$ \\
ATCA & 31 & 22 & $14.0^{\prime}$ & $19.7^{\prime}$ \\
\hline
\end{tabular}

Table 2: Largest Angular Sizes (LAS) of different synthesis telescopes. Given for the different telescopes (1) are: minimum baseline (2), antenna diameter (3), LAS from the minimum physical baseline (4), and LAS down to the shadowing limit (5).

sion and were insensitive to the large scale $\left(>15^{\prime}\right)$ low column density extra planar HI.

In theory, the Largest Angular Sizes (LAS) accessible to an aperture synthesis telescope is given by:

$$
\mathrm{LAS}=0.6 \times \lambda / \mathrm{B}_{\min }
$$

When trying to observe LAS, Table 2 illustrates why KAT-7 should be a good instrument for this purpose. If we consider the shortest physical baseline, it can be seen that the gain of KAT-7 is $\sim 33 \%$ over the VLA, WSRT (in its Maxishort configuration) and ATCA. In fact, the minimum projected baseline is the antenna diameter. This means that if we instead consider the shadowing limit, the gain over the VLA, WSRT and ATCA is a factor of $2\left(\sim 36^{\prime}\right.$ vs $\left.18^{\prime}\right)$.

In practice, the largest scale that can be reliably imaged cannot be assessed as simply as Table 2 suggests. When less flux is recovered with an interferometer than with a single dish, the shortcoming is often explained by a lack of short spacings. Yet it may often be the case that additional care in calibration and imaging can resolve the discrepancy. The resulting quality when imaging large-scale emission is critically dependent on several factors including the image reconstruction techniques that are employed (classic CLEAN, MEM, multi-resolution CLEAN, etc). It is important to note that the spatial scales of interest should be assessed per spectral channel, not for the entire galaxy. In single channels, the angular scales are always more restricted. A good illustration of imaging structures larger than the nominal LAS is the study of NGC 628 by Kamphuis \& Briggs (1992), in which features as large as $\sim 25^{\prime}$ are seen in individual channels (see their Fig. 1 showing the naturally weighted channel maps), although emission on scales larger than $\sim 17.5$ 'should not be seen by the VLA in D configuration. Nevertheless, it remains true that KAT-7's smaller dish diameter and shorter baselines enable it to image larger structures than with the VLA, WSRT and ATCA.

The remainder of this paper is as follow. In Sec. 2, a description of the KAT-7 observations and data reduction will be given. Sec. 3 will discuss the continuum emission and Sec. 4 will describe the HI content and distribution. Sec. 5 will explain how to distinguish between the HI in the disk and the $\mathrm{HI}$ in the halo while Sec. 6 will study the HI kinematics of both components. The main results will be discussed in Sec. 7 and, finally, a summary and final conclusions will be given in Sec. 8 . 

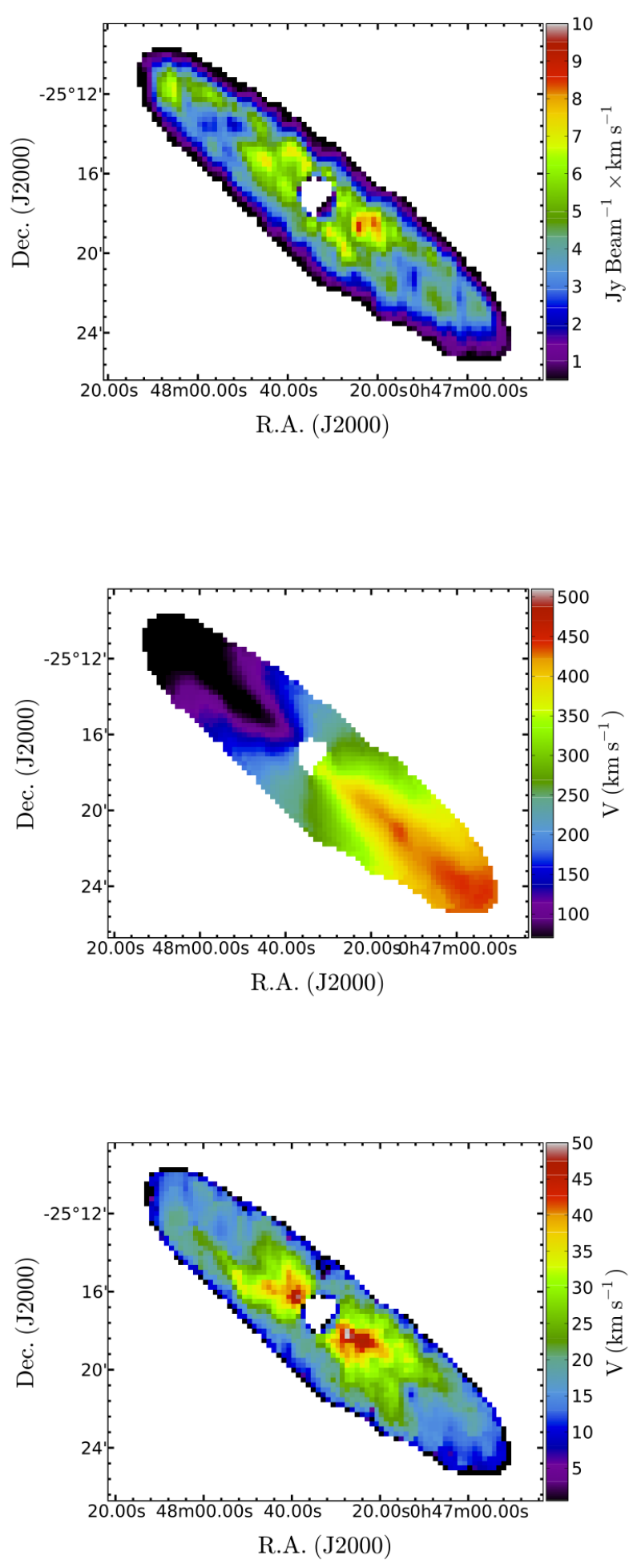

Figure 3. VLA moment maps (top: Hi distribution; middle: velocity field; bottom: velocity dispersion) of NGC 253 derived from the data set presented by (Puche, Carignan \& van Gorkom 1991). The velocity resolution is $20.6 \mathrm{~km} \mathrm{~s}^{-1}$ and the spatial resolution is $68^{\prime \prime} \times 68^{\prime \prime}$.

\begin{tabular}{lr}
\hline \hline Parameter & Value \\
\hline Number of antennas & 7 \\
Dish diameter & $12 \mathrm{~m}$ \\
Min baseline & $26 \mathrm{~m}$ \\
Max baseline & $185 \mathrm{~m}$ \\
Frequency range & $1200-1950 \mathrm{MHz}$ \\
Max instantaneous bandwidth & $256 \mathrm{MHz}$ \\
Polarisation & Linear H \& V \\
$\mathrm{T}_{\text {sys }}$ & $26 \mathrm{~K}$ \\
Aperture efficiency & 0.65 \\
System Equivalent Flux Density & $1000 \mathrm{Jy}$ \\
Latitude & $-30: 43: 17.34$ \\
Longitude & $21: 24: 38.46$ \\
Elevation & $1038 \mathrm{~m}$ \\
Digital back-end & ROACH boards \\
\hline
\end{tabular}

Table 3: KAT-7 specifications.

\begin{tabular}{lccc}
\hline \hline mode & $\begin{array}{c}\text { total BW } \\
(\mathrm{MHz})\end{array}$ & $\begin{array}{c}\text { number of } \\
\text { channels }\end{array}$ & $\begin{array}{c}\text { channel BW } \\
(\mathrm{kHz})\end{array}$ \\
\hline c16n2M4k & 1.5625 & 4096 & 0.381 \\
c16n7M4k & 6.25 & 4096 & 1.526 \\
c16n25M4k & 25 & 4096 & 6.104 \\
c16n400M4k & 256 & 1024 & 390.625 \\
\hline
\end{tabular}

Table 4: KAT-7 correlator modes.

\section{KAT-7 OBSERVATIONS AND DATA REDUCTION}

The KAT-7 dishes have a prime-focus alt-az design with a F/D of 0.38 , optimized for single-pixel L-band feeds. The low noise amplifiers (LNAs) for the feeds are cryogenically cooled to $80 \mathrm{~K}$ using Stirling coolers. The key system specifications for KAT-7 are summarized in Table 3. The digital backend of the system uses the Reconfigurable Open Architecture Computing Hardware (ROACHhttps://casper.berkeley.edu/wiki/ROACH), which is a flexible and scalable system enabling spectral line modes covering a wide range of resolutions. Table 4 gives the details of the recently commissioned correlator modes. Digital filters give a flat bandpass over the inner $75 \%$ of the band with a rapid roll-off at the edges of the band.

The parameters of the KAT-7 observations of NGC 253 are given in Table 5. The data were collected over 17 observing sessions between 2013 March and 2013 July using the c16n25M4K spectral line mode. This correlator mode gives velocity channels of $1.3 \mathrm{~km} \mathrm{~s}^{-1}$ over a flat bandpass of $4000 \mathrm{~km} \mathrm{~s}^{-1}$. The larger bandwidth was used to look for background galaxies in the field and to ensure a good estimation of the continuum for subtraction. All antennas were in the array during all the observing sessions. The median time of each session on target was 9 hours for a total project time of 153.5 hours, including calibration and slew time. Total time on NGC 253 was 115.6 hours.

Each of the seventeen observing sessions were reduced separately. All data calibration was done using standard calibration tasks in the Common Astronomy Software Applications (CASA 3.4.0) package (McMullin et al. 2007). The KAT-7 primary beam is large enough to observe NGC 253 in a single pointing. Phase drifts as a function of time were corrected by means of a nearby point source (0023-263) observed every ten minutes. This source 


\begin{tabular}{lr}
\hline \hline Parameter & Value \\
\hline Start of observations & 5 March 2013 \\
End of observations & 19 July 2013 \\
Total integration (on source) & 115.6 hours \\
FWHM of primary beam & $1.08^{\circ}$ \\
Total Bandwidth & $25 \mathrm{MHz}$ \\
Central frequency & $1419 \mathrm{MHz}$ \\
Channel Bandwidth & $6.1 \mathrm{kHz}$ \\
Number of channels & 4096 \\
Channel width & $1.28 \mathrm{~km} \mathrm{~s}-1$ \\
Maps gridding & $20^{\prime \prime} \times 20^{\prime \prime}$ \\
Maps size & $257 \times 257$ \\
Flux calibrator & $3 \mathrm{C} 138$ \\
Phase/bandpass calibrator & $0023-263$ \\
\hline Robust $=0$ weighting function & $213^{\prime \prime} \times 188^{\prime \prime}$ \\
FWHM of synthesized beam & $1.0 \mathrm{mJy} / \mathrm{beam}^{-2}$ \\
RMS noise & $1.3 \times 10^{19} \mathrm{~cm}^{-2}$ \\
\hline Column density limit $(3 \sigma)$ &
\end{tabular}

Table 5: Parameters of the KAT-7 observations.

was also used to correct for variations in the gain as a function of frequency (bandpass calibration). The absolute flux scale was set by observations of $3 \mathrm{C} 138$. Comparisons of the flux measurements on the observed calibrators suggests that the absolute flux uncertainties are on the order of $5 \%$. Variations in the bandpass are on the order of $1 \%$.

Initial imaging revealed which channels were free of HI emission. Continuum emission was subtracted from the raw UV data by making first order fits to the line free channels using the CASA task UVCONTSUB. The calibration was then applied and NGC 253 was then SPLIT from the calibration sources. In order to make sure no residual calibration errors remained in the data, a test cube was made using the CASA task CLEAN. KAT-7 does not employ doppler tracking and CASA does not fully recognize frequency keywords, so special care was taken to produce uv data sets and test cubes with the proper velocity coordinates (see Carignan et al. 2013). The individual calibrated continuum subtracted uv data sets were then combined together using the CASA task CONCAT.

Preliminary imaging of the combined data in CASA revealed the presence of artifacts in the form of horizontal lines which we were able to identify as the accumulation of RFI at $u=0$. This is most likely due to the fact that for those visibilities the fringe rotation is zero. These were removed by flagging all visibilities near $\mathrm{u}=$ 0 . This uncovered further artifacts in the form of diagonal lines parallel to the major axis of NGC 253. To remove this problem, it was found necessary to use the task SELFCAL in Miriad (Sault, Teuben \& Wright 1995), on the strong continuum of NGC 253. This removed most of these artifacts giving a final rms of $1 \mathrm{mJy} / \mathrm{beam}$ with channels smoothed to $5 \mathrm{~km} \mathrm{~s}^{-1}$ in the final cube.

\section{CONTINUUM EMISSION}

Figure 4 shows the KAT-7 continuum map of NGC 253. The total flux at $1.419 \mathrm{GHz}$ is $6.8 \pm 0.3 \mathrm{Jy}$. This is comparable with the previous measurements of $6.5 \pm 0.6 \mathrm{Jy}$ at $1.415 \mathrm{GHz}$ (de la Beaujardière et al. 1968) and $6.7 \pm 0.7 \mathrm{Jy}$ at $1.430 \mathrm{GHz}$ (Heidmann 1963). When comparing Fig. 4 to the observations at $1.46 \mathrm{GHz}$ of
Hummel, Smith \& van der Hulst (1984) or at $1.49 \mathrm{GHz}$ of Condon (1987), the emission is slightly more extended ( 20\%) along the major axis but $60 \%$ more along the minor axis (see also Carilli et al. 1992). In fact, the emission reaches the very edge of the optical disc on this very deep image from Malin \& Hadley (1997). However, the difference between our continuum map and the one of Hummel, Smith \& van der Hulst (1984) is not surprising since they themselves mentioned that their emission is sitting in the middle of a negative bowl, a signature of the lack of short enough baselines at the VLA.

Looking at the very high flux in the centre, it is clear that the starburst is responsible for a large part of the emission, while the spiral arms are more responsible for the lower flux in the outer disk. Along the major axis, it can be seen that the emission stops at the end of the spiral arms. Contrary to what is seen in the observations of some edge-on galaxies, where the axis ratio of the continuum emission is similar to the axis ratio of the optical disk (Hummel, Sancisi \& Ekers 1984), the axis ratio is twice that of the optical disk ( 0.50 vs 0.25$)$ in the case of NGC 253.

However, NGC 253 is not the only exception. NGC 4631 (Ekers \& Sancisi 1977), which has strong X-ray emission originating from the nucleus and is interacting with a dwarf companion along the minor axis, also has a much thicker radio disk. This is only beaten by NGC 253's twin M82 (Seaquist \& Odegard 1991), which has an axis ratio $\sim 1.0$ (vs optical $\sim 0.4$ ). Higher resolution (VLA, A configuration) $20 \mathrm{~cm}$ observations of the centre of NGC 253 (U1vestad \& Antonucci 1997) show an even thicker disk with an axis ratio $\sim 0.65$.

Allen, Baldwin \& Sancisi (1978) explain that the sources of relativistic electrons are distributed like the light in the optical disk and that the electrons diffuse outwards. However, the larger thickness of the radio disk in this case suggests that the starburst may be responsible for an extra z-component of the magnetic field, which enhances the propagation of the relativistic electrons in the direction perpendicular to the plane. As discussed by Heald (2012), a substantial vertical displacement of an initially plane-parallelordered magnetic field may be driven by a star formation event. A detailed study of the magnetic field in the nuclear outflow of NGC 253 can be found in Heesen et al. (2011).

Since the KAT-7 observations were carried out over several months, we have a unique opportunity to investigate whether or not the continuum in the central regions exhibits time variability. To this end we calculate the continuum flux inside a $2^{\prime}$ radius covering the absorption feature for all seventeen separate data sets. No significant variation in continuum flux is observed. All measured fluxes are within the measurement uncertainties $(\sim 5 \%)$. It should be noted here again that the bulk of the radio continuum comes from the central starburst, reducing the likelihood of detecting significant variations in continuum flux.

\section{HI CONTENT AND DISTRIBUTION}

The total HI distribution map, shown in Figure 5, was derived using the task MOMNT (moment 0) in AIPS (Greisen 2003). It is superposed on a composite image of FUV from Galex, IR from WISE and soft X-rays from ROSAT (0.1-0.4 keV). The faintest level goes down to $\sim 1.0 \times 10^{19} \mathrm{~cm}^{-2}$. At that level, the galaxy has a diameter of $34 \pm 2 \mathrm{kpc}$ (the error being defined by the beam size), which is comparable to the optical diameter (cf. Tab. 1). It can be seen that a significant fraction of the HI can be found away from the plane out to projected distances of $\sim 9-10 \mathrm{kpc}$ in the centre and 13-14 kpc 


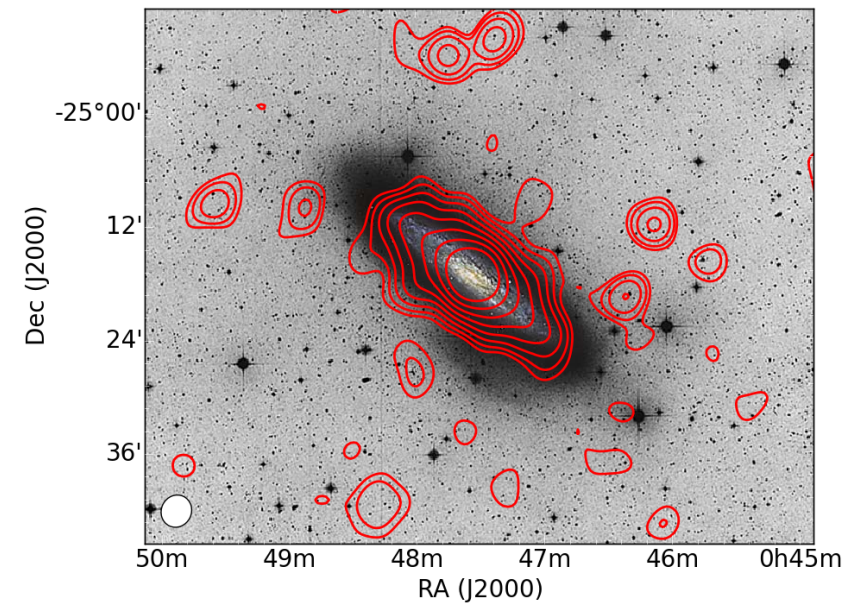

Figure 4. Continuum map of NGC 253, overlaid on a deep optical image (Malin \& Hadley 1997). The contours are $7.5(3 \sigma), 15,30,60,120,240$, $480,960 \mathrm{mJy} / \mathrm{beam}$. The beam is in the bottom left corner.

at the edge of the disk, which is comparable to the extent of the extra planar emission seen in the edge-on spiral NGC 891 (Oosterloo, Fraternali \& Sancisi 2007). Looking at Fig. 5, the HI appears to surround the hot gas component in the outer parts.

The global HI profile of NGC 253 is given in Figure 6. This was obtained using the task BLSUM in AIPS after primary beam correction. The asymmetry is clear with more $\mathrm{HI}$ on the receding (SW) than on the approaching side. From it, a mid-point velocity of $242 \pm 4 \mathrm{~km} \mathrm{~s}^{-1}$ (50\% level) is found. Because of the asymmetry, this value has to be preferred to the intensity weighted mean velocity as indicative of the systemic velocity. This can be compared to $243 \pm 2 \mathrm{~km} \mathrm{~s}^{-1}$ found by the HIPASS survey (Koribalski et al 2004). However, for the kinematical analysis of Sec. 6, we will use the kinematically determined value. The profile widths at the $20 \%$ and $50 \%$ levels are $\Delta \mathrm{V}_{20}=439 \pm 4 \mathrm{~km} \mathrm{~s}^{-1}$ and $\Delta \mathrm{V}_{50}=412 \pm 4$ $\mathrm{km} \mathrm{s}^{-1}$, slightly larger than the Koribalski et al (2004) values. A total Hi flux of $728 \pm 36 \mathrm{Jy} . \mathrm{km} \mathrm{s}^{-1}$ is found (after primary beam correction), which corresponds to an Hi mass of $2.1 \pm 0.1 \times 10^{9}$ $\mathrm{M}_{\odot}$ for a $\mathrm{M}_{\mathrm{HI}} / \mathrm{L}_{\mathrm{B}} \simeq 0.1$ at our adopted distance of $3.5 \mathrm{Mpc}$. This is similar to the mass of $2.0 \times 10^{9} \mathrm{M}_{\odot}$ found by Boomsma et al. (2005). Note that in the next section, we will separate the disk and the kinematically anomalous (halo) components. The mass of the anomalous gas is given in Table 6.

The radial HI profile of NGC 253 is given in Figure 7 and compared to the profile derived by the previous VLA observations. It can be seen that, while the VLA was detecting the bright HI disk (down to $2.4 \times 10^{20} \mathrm{~cm}^{-2}$ ), KAT-7 detects $\sim 33 \%$ more flux (down to $1.3 \times 10^{19} \mathrm{~cm}^{-2}$ ), mainly in the outer disk and in the halo. This is not only coming from the longer integration but also because KAT-7 can detect scales larger than $15^{\prime}$, scales invisible to the VLA because of the lack of short spacings.

The derived Hi parameters are summarized in Table 6. While the HI disk of a late-type spiral is often much more extended than the stellar disk $\left(D_{25}\right)$, this is not the case for NGC 253. At a level of $1 \mathrm{M}_{\odot} \mathrm{pc}^{-2}\left(1.25 \times 10^{20} \mathrm{~cm}^{-2}\right)$, the HI disk of NGC 253 is equivalent to the stellar disk (isophotal major diameter, $D_{25}$ ) and even at a level of $10^{19} \mathrm{~cm}^{-2}$, it is only equivalent to the Holmberg diameter $\left(D_{H O}\right.$ : diameter at a surface brightness level of $\mu_{B}=26.6$ mag $\left.\operatorname{arcsec}^{-2}\right)$. For example, NGC 300, another late-type spiral in the Sculptor group, has an HI disk 50\% larger than the optical disk (Puche, Carignan \& Bosma 1990). What is exceptional with

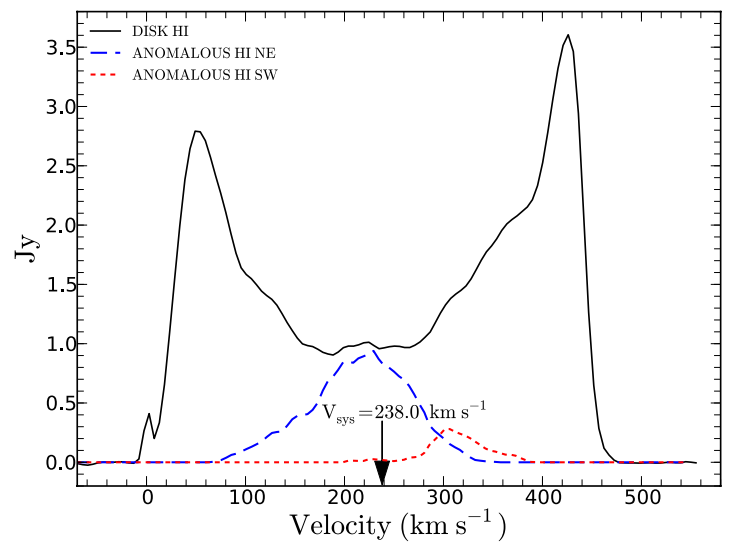

Figure 6. Global profile (black) for the KAT-7 HI observations with the kinematical $\mathrm{V}_{\text {sys }}$ indicated (see Sec. 6). Also plotted are the HI profiles for the receding (red dotted) and approaching (blue dashed) components of the Halo (see Sec. 5). The channel width is $5 \mathrm{~km} \mathrm{~s}^{-1}$.

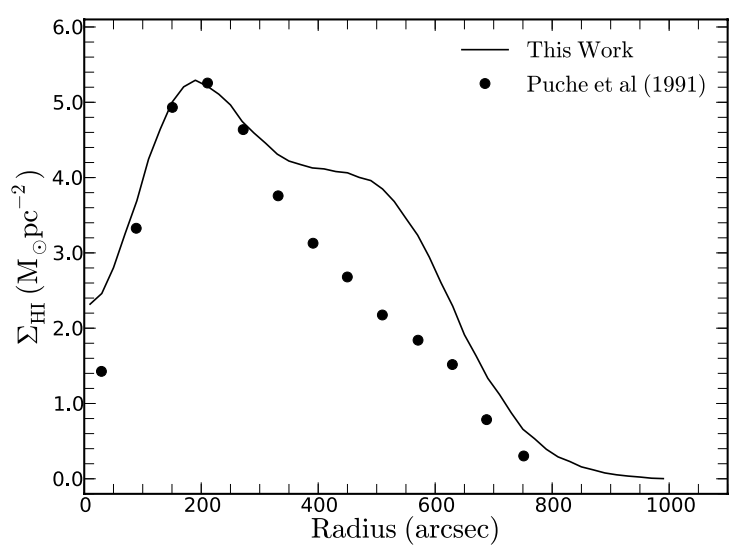

Figure 7. Comparison of the VLA (Puche, Carignan \& van Gorkom 1991) and the KAT-7 HI radial profiles.

NGC 253 is not its radial extent but more its extent perpendicular to the disk. Despite the apparent truncation of the HI disk, NGC 253 is not HI deficient with $\log \left(\mathrm{M}_{\mathrm{HI}}\right)=9.32$, slightly larger than $<\log \left(\mathrm{M}_{\mathrm{HI}}\right)>$ for an isolated field Sc of 9.24 (Solanes, Giovanelli \& Haynes 1996).

\section{SEPARATING THE DISK AND HALO HI GAS}

The HI total intensity map of NGC 253 clearly shows some of the HI to be spatially separated from the main disk. Furthermore, the kinematics of this spatially extended HI component differ from that of the main disk. Position-Velocity (PV) slices, extracted parallel to the major axis of NGC 253, provide clear evidence of kinematically anomalous HI. Such a PV slice, passing through the centre of the galaxy, is shown in Figure 8. For the approaching (NE) half of the galaxy, it clearly highlights the presence of an anomalous HI component spanning a range of radial velocities that is offset from the high-intensity emission of the main disk; toward the systemic velocity of the system. Such anomalous Hi features are commonly seen in deep observations of nearby galaxies: e.g. NGC 891 (Oost- 


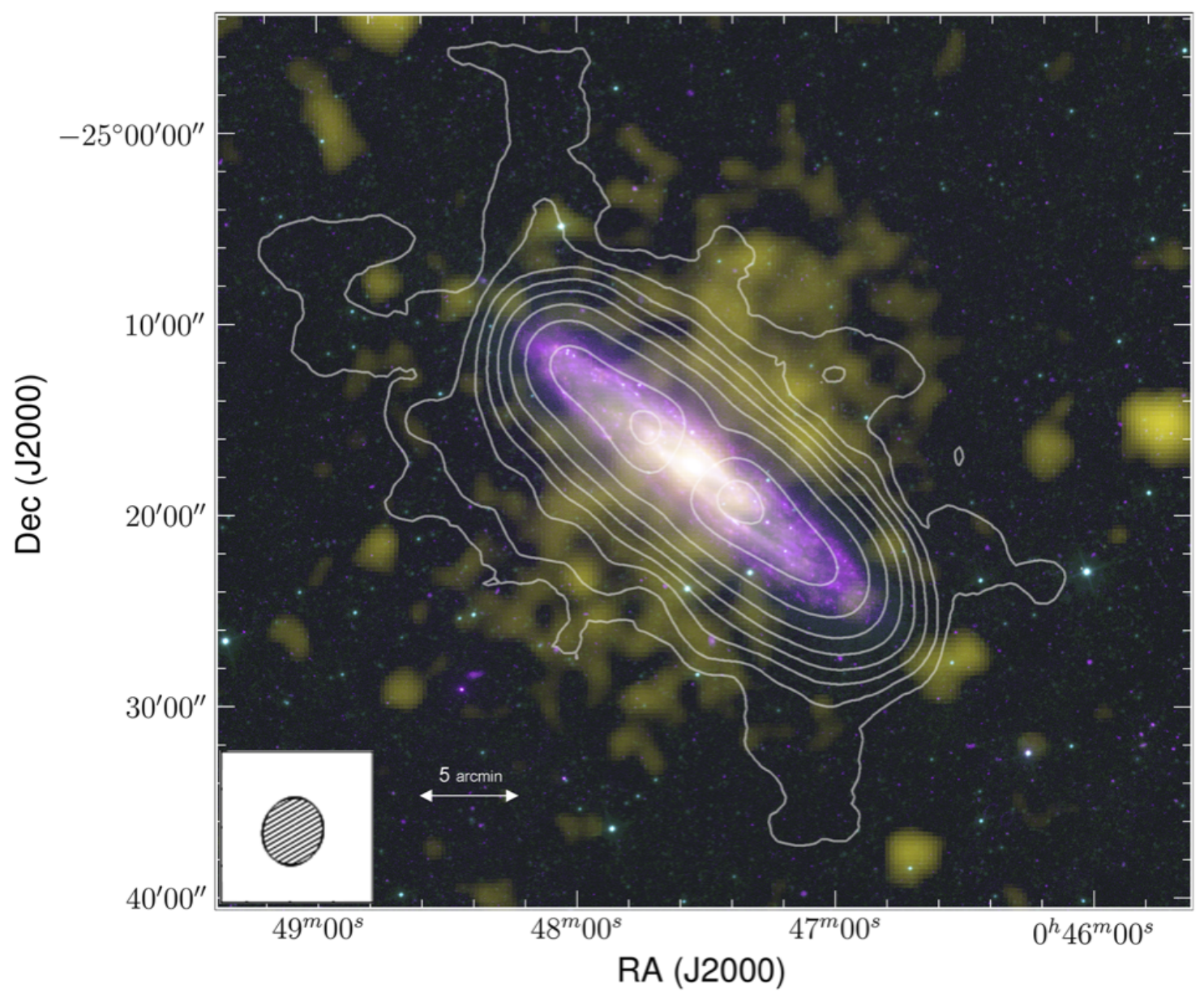

Figure 5. Combined WISE (RGB) + FUV (magenta) + X-rays (yellow: Rosat) + HI contours of the moment 0 map (scale: $1^{\prime} \sim 1 \mathrm{kpc}$ ). The contours correspond to $1.3,2.6,5.2,10.4,20.8,41.6,83.2,166.4 \& 253.3 \times 10^{19} \mathrm{~cm}^{-2}$. The ROSAT data are from Pietsch et al. (2000).

\begin{tabular}{lc}
\hline \hline Parameter & Value \\
\hline $\mathrm{M}_{\mathrm{HI}}$ (total) & $2.1 \pm 0.1 \times 10^{9} \mathrm{M}_{\odot}$ \\
$\mathrm{M}_{\mathrm{HI}}$ (anom.) $)^{1}$ & $7.8 \times 10^{7} \mathrm{M}_{\odot}$ \\
$\mathrm{M}_{\mathrm{HI}}$ (anom.)/M $/ \mathrm{M}_{\mathrm{HI}}$ (total) $)^{1}$ & $3.5 \%$ \\
$\Delta \mathrm{V}_{20},(20 \%$ level) & $439 \pm 4 \mathrm{~km} \mathrm{~s}^{-1}$ \\
$\Delta \mathrm{V}_{50},(50 \%$ level) & $412 \pm 4 \mathrm{~km} \mathrm{~s}^{-1}$ \\
$\mathrm{~V}_{\text {sys }}$ (mid-point 50\%) & $242 \pm 4 \mathrm{~km} \mathrm{~s}^{-1}$ \\
$\mathrm{~V}_{\text {sys }}$ (kinematical, TR) & $238 \pm 4 \mathrm{~km} \mathrm{~s}^{-1}$ \\
$\mathrm{D}_{20},\left(\right.$ diam. at $10^{20} \mathrm{~cm}^{-2}$ level) & $29 \pm 2 \mathrm{kpc}$ \\
$\mathrm{D}_{19},\left(\right.$ diam. at $10^{19} \mathrm{~cm}^{-2}$ level) & $34 \pm 2 \mathrm{kpc}$ \\
\hline
\end{tabular}

(1) These are lower limits.

Table 6: HI parameters of NGC 253.

erloo, Fraternali \& Sancisi 2007) and NGC 2403 (Fraternali et al. 2001).

In order to isolate the anomalous HI emission in NGC 253 from the regular $\mathrm{HI}$, PV slices aligned with the major axis were extracted along the full extent of the galaxy's minor axis. Each PV slice was visually inspected. Any emission deemed to be kinematically anomalous was isolated, removed, and used to construct a new cube. In Figure 8, the anomalous HI is delimited by the white con- tours. This position-velocity diagram is representative of the majority of PV slices with anomalous HI.

The spatial distribution of the anomalous HI in that new cube is delimited by red contours in the channel maps shown in Figure 9. Summing all the gas gives an extended component on the approaching half of the galaxy and a smaller component on the receding side as can be seen in Figure 10. The mass of that component is estimated to be $\sim 3.5 \%$ of the total HI mass of the galaxy $\left(\sim 7.8 \times 10^{7} \mathrm{M}_{\odot}\right)$. This is equivalent to the mass of the anomalous gas found by Boomsma et al. (2005).

Naturally, this should be seen as a lower limit since our technique has most probably missed a large fraction of the "anomalous" gas, especially along the minor axis to the NW and SE due to projection effects. This can be seen on the PV slices along the minor axis shown in Figure 11. However, this will not be a problem for the kinematical study since, as we will see in Sec. 6, the data along the minor axis will be excluded.

In order to generate a velocity field representative of the circular kinematics of the HI disk, we again used PV slices aligned with the major axis of the galaxy, and fitted interactively three Gaussians to all of the line profiles making up a slice. Great care was taken in selecting the Gaussian associated with the disk of the galaxy. This was done by simultaneously viewing a position velocity slice and the fitted Gaussians. The line profile along the white dashed line on the left part of Figure 8 is shown on the right. For this particular 

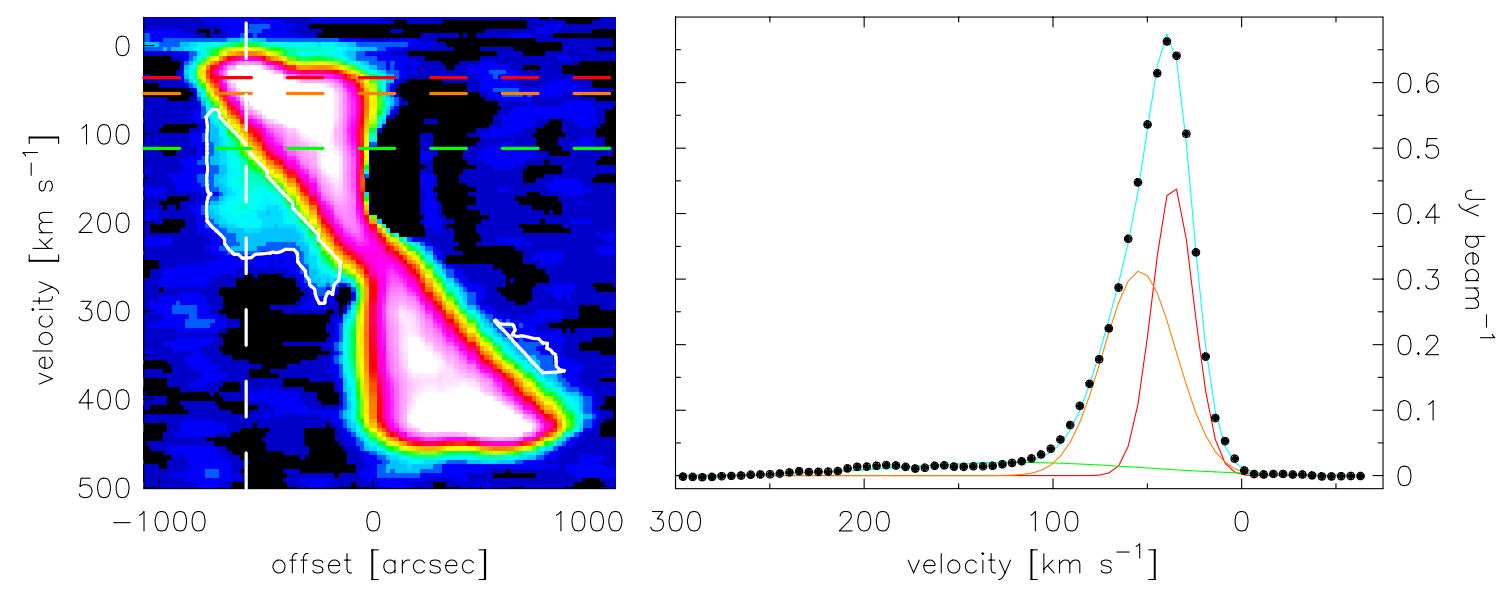

Figure 8. (left) PV slice through the centre of the galaxy. The velocities corresponding to the peaks of the Gaussians have been indicated with the horizontal dashed lines, while the vertical dashed white line shows the location of the profile on the right. (right) The red Gaussian traces the HI ridge, the orange Gaussian traces the beam-smeared emission, and the green Gaussian is fitting the anomalous HI.

line profile, the red Gaussian parameterizes the radial velocity of the high-intensity $\mathrm{HI}$ ridge in the PV slice. This is the component of the line profile associated with the regularly-rotating Hi disk of the galaxy. The orange Gaussian, with its peak shifted toward systemic velocity, represents the beam-smeared component of the line profile. The green Gaussian captures the kinematically anomalous HI emission.

Stepping along the minor axis of the galaxy, this procedure was repeated for all the PV slices aligned parallel to the major axis. In doing so, a velocity field representing the circular kinematics of the HI disk of NGC 253 was built up pixel-by-pixel. When none of the Gaussians fitted to a line profile provided a reliable measure of the circular rotation, a blank was assigned to the corresponding position in the velocity field. We consider the velocity field shown in Figure 12 (top panel) to better represent the circular kinematics of NGC 253 than the traditional intensity-weighted-mean velocity field. From the cube built for the anomalous HI, a velocity field was also obtained by a moment analysis and is shown in the bottom panel of Figure 12.

\section{HI KINEMATICS OF THE DISK AND HALO GAS}

To derive the RCs, we used the implementation of the tilted ring model (Rogstad, Lockhart \& Wright 1974) in the GIPSY (van der Hulst et al. 1992) task ROTCUR (Begeman 1989, 1987). In the tilted ring model, a set of concentric rings is used to describe the motion of the gas in the galaxy. The gas is assumed to be in circular motion. Each ring is characterized by a set of 5 orientation parameters, namely: a rotation centre $\left(x_{c}, y_{c}\right)$, a systemic velocity $V_{s y s}$, an inclination $i$, a Position Angle $P A$ and by a rotation velocity $V_{C}$. Naturally, the rotation centre $\left(x_{c}, y_{c}\right)$ and the systemic velocity $V_{\text {sys }}$ should be the same for all the rings, at least within the optical disk, but $i$ and $P A$ will vary if the HI disk is warped outside $\mathrm{D}_{25}$.

The line of sight velocity at any $(x, y)$ position in a ring with radius $R$ is given by

$$
V(x, y)=V_{\text {sys }}+V_{C} \sin (i) \cos (\theta)
$$

where $\theta$ is the position angle with respect to the receding major axis

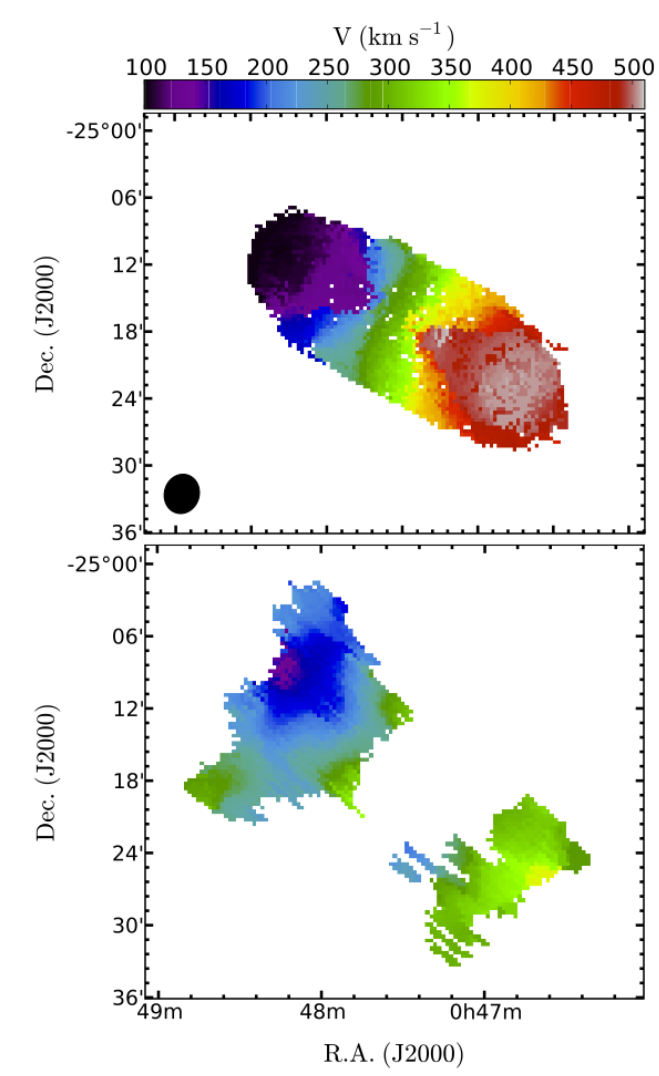

Figure 12. Velocity fields of the disk (top) and anomalous (bottom) HI.

measured in the plane of the galaxy. $\theta$ is related to the actual $P A$ in the plane of the sky by

$$
\begin{aligned}
& \cos (\theta)=\frac{-\left(x-x_{0}\right) \sin (P A)+\left(y-y_{0}\right) \cos (P A)}{R} \\
& \sin (\theta)=\frac{-\left(x-x_{0}\right) \cos (P A)+\left(y-y_{0}\right) \cos (P A)}{R \cos (i)}
\end{aligned}
$$




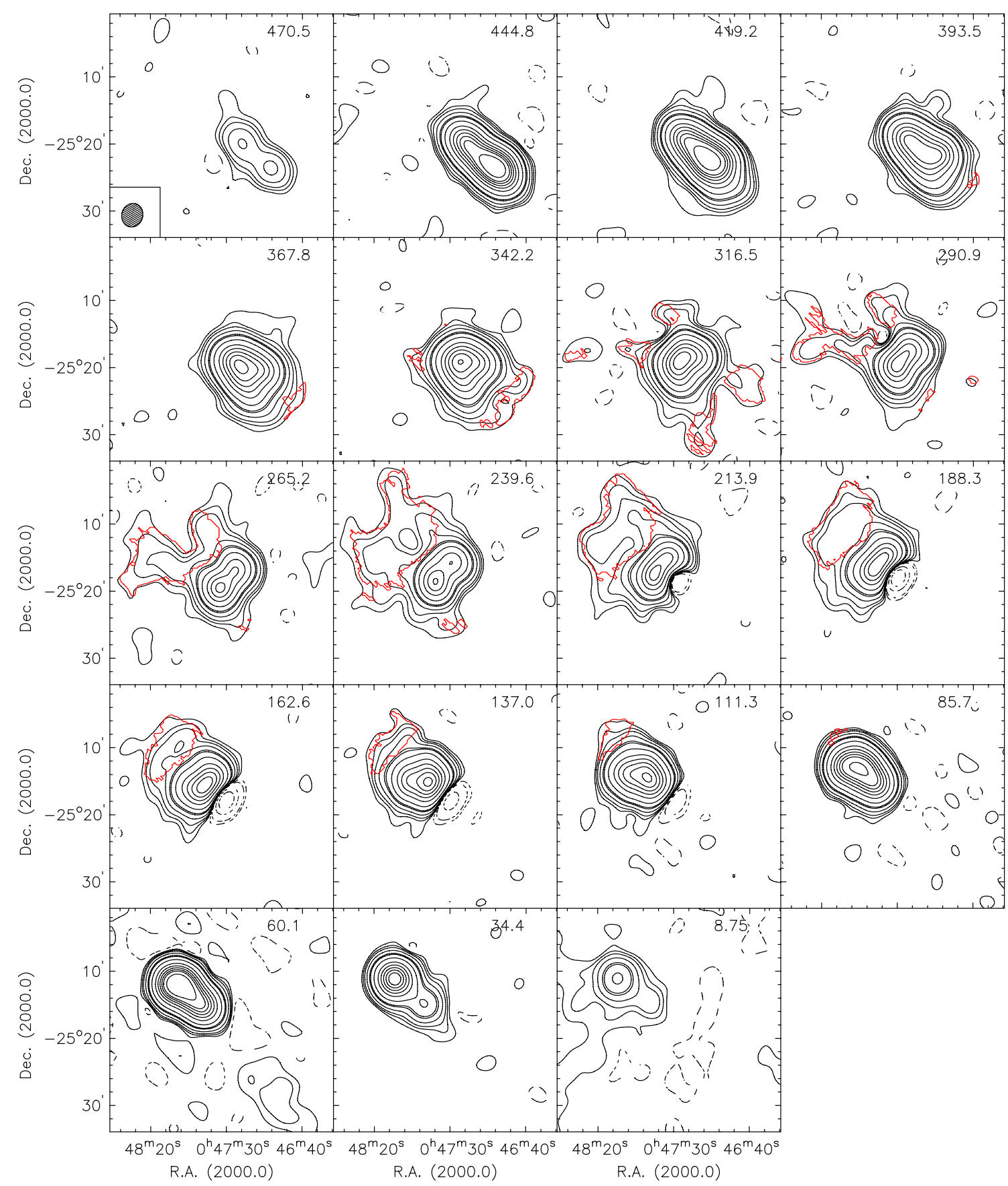

Figure 9. Individual channel maps identifying the anomalous HI emission. The solid black contours are at levels of 0.80 .60 .50 .40 .3 $0.20 .10 .050 .040 .020 .010 .0060 .003 \mathrm{Jy} / \mathrm{beam}$. The dashed black contours are at $-0.1-0.05-0.01-0.003 \mathrm{Jy} / \mathrm{beam}$. The red contour is at $0.003 \mathrm{Jy} / \mathrm{beam}$. The beam is shown in the bottom left corner of the first channel.

\subsection{Tilted-ring model for the HI disk}

A $|\cos \theta|^{2}$ weighting function and an exclusion angle of $60^{\circ}$ about the minor axis have been used to give maximum weight to the velocity points close to the major axis and to minimize the influence of large deprojection errors close to the minor axis in view of the large inclination of the galaxy. We used two rings per beam size.

The method consists of finding for each ring the best set of the 5 orientation parameters $\left(x_{c}, y_{c}\right), V_{s y s}, i$ and $P A$ which minimizes the dispersion of $V_{C}$ inside the ring. The following procedure is used:
- The rotation center $\left(x_{c}, y_{c}\right)$ and the systemic velocity $V_{s y s}$ are determined first by keeping $i$ and $P A$ fixed (using the optical values). The rotation center and the systemic velocity have to be determined simultaneously since they are correlated.

- Now, keeping $\left(x_{c}, y_{c}\right)$ and $V_{s y s}$ fixed, $i$ and $P A$ are fitted to map any possible warp of the HI disk. The warps usually start just outside the optical. However, since the HI disk in NGC 253 is about the same size as the optical, we may not reach the warp region. Here also, $i$ and $P A$ have to be determined simultaneously since they are correlated. 


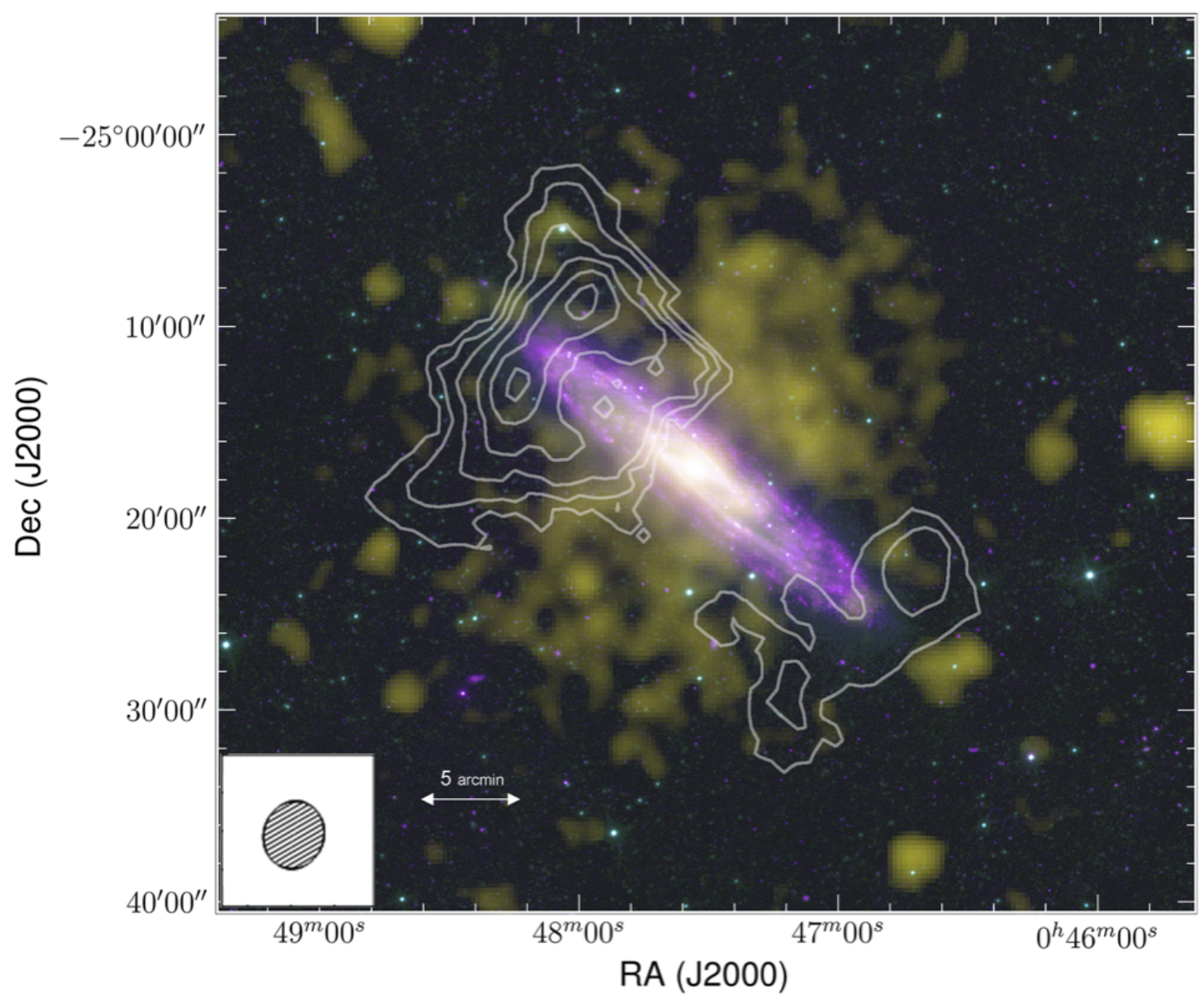

Figure 10. Combined WISE (RGB) + FUV (magenta) + X-rays (yellow: Rosat) + HI contours of the anomalous HI (scale: $\left.1^{\prime} \sim 1 \mathrm{kpc}\right)$. The contours correspond to $0.03,0.2,0.5,1.0,1.52 .0 \mathrm{Jy} /$ beam $\mathrm{km} \mathrm{s}^{-1}$.

- The previous two steps were done using the data of both sides of the galaxy together. Using the same fixed $\left(x_{c}, y_{c}\right)$ and $V_{s y s}$, the previous step is repeated for the approaching and receding sides separately to identify possible departures from axisymmetry.

The ROTCUR solutions (approaching, receding, both sides) are shown in Figure 13 with $P A$ and $i$ free to vary. Because of the low resolution of our data, it can be seen that, while $P A$ is fairly well determined $(<P A\rangle=235^{\circ} \pm 5^{\circ}, i$ is not constrained at all. It is clear, looking at the galaxy, that inclinations $\sim 40^{\circ}$, as seen for $r>10^{\prime}$, are completely unrealistic. However, much higher resolution data $\left(30^{\prime \prime}\right)$ exist from Fabry-Perot observations (Hlavacek-Larrondo et al. 2011). In their Figure 10, we can see that the inclination is nearly constant at $i=76^{\circ} \pm 4^{\circ}$. Additionally, similar values of $\mathrm{i}\left(78.5^{\circ}\right)$ and PA $\left(232^{\circ}\right)$ have been inferred from CO observations (e.g. Sofue et al. 1999; Hougton et al. 1997). In the case of NGC 253, adopting constant $P A$ and $i$ is reasonable since, as we have seen in Sec. 4, the HI disk has the same size as the optical disk and warps usually start past the optical radius. We thus adopt constant values of $P A=235^{\circ}$ and $i=76^{\circ}$ (green lines in Fig. 13) to derive our adopted RC.

A similar approach was used by Puche, Carignan \& van Gorkom (1991) using higher resolution HI data. The adopted RC for the HI disk (solution for both sides with $P A$ and $i$ fixed) is given in Table 7. An important point to notice is the large errors on the first two points in the inner parts. This is expected for at least three reasons: 1 - the strong absorption feature in the centre; 2- the small quantity of $\mathrm{HI}$ in the centre (cf. Fig, 7); 3- the strong disturbance expected from the starburst in the inner regions.

\subsubsection{Three-dimensional modelling}

As a further test on our choice of $i$, three different tilted ring models were generated, with constant inclinations of 66, 76 and 86 degrees. For each of the models, all the other parameters $\left(P A, V_{s y s}, X_{c}, Y_{c}\right)$ were kept fixed to the same constant values, allowing only $V_{c}$ to vary with radius. The tilted ring models were then used to generate $3 \mathrm{D}$ models of the NGC $253 \mathrm{HI}$ data cube. This was done using the task GALMOD in GIPSY. For the radial distribution of HI we adopted the HI surface density profile shown in Figure 7. A fiducial HI disk scale height of $0.5 \mathrm{kpc}$ was used for each model. The resulting model cubes were smoothed to the spatial resolution of the data, and the fluxes scaled based on the NGC 253's integrated flux density of $728 \mathrm{Jy} \mathrm{km} / \mathrm{s}$.

These models, each based on a different inclination, were then compared to the data on a channel-by-channel basis. Doing so clearly showed that the $i=76^{\circ}$ model consistently matches the data better than the models with $i=66^{\circ}$ and $i=86^{\circ}$. For three different channels, Figure 14 shows the comparisons between the models and the data. Clearly, $i=66^{\circ}$ (left) produces emission too elongated in the minor axis direction and $i=86^{\circ}$ (right) too nar- 

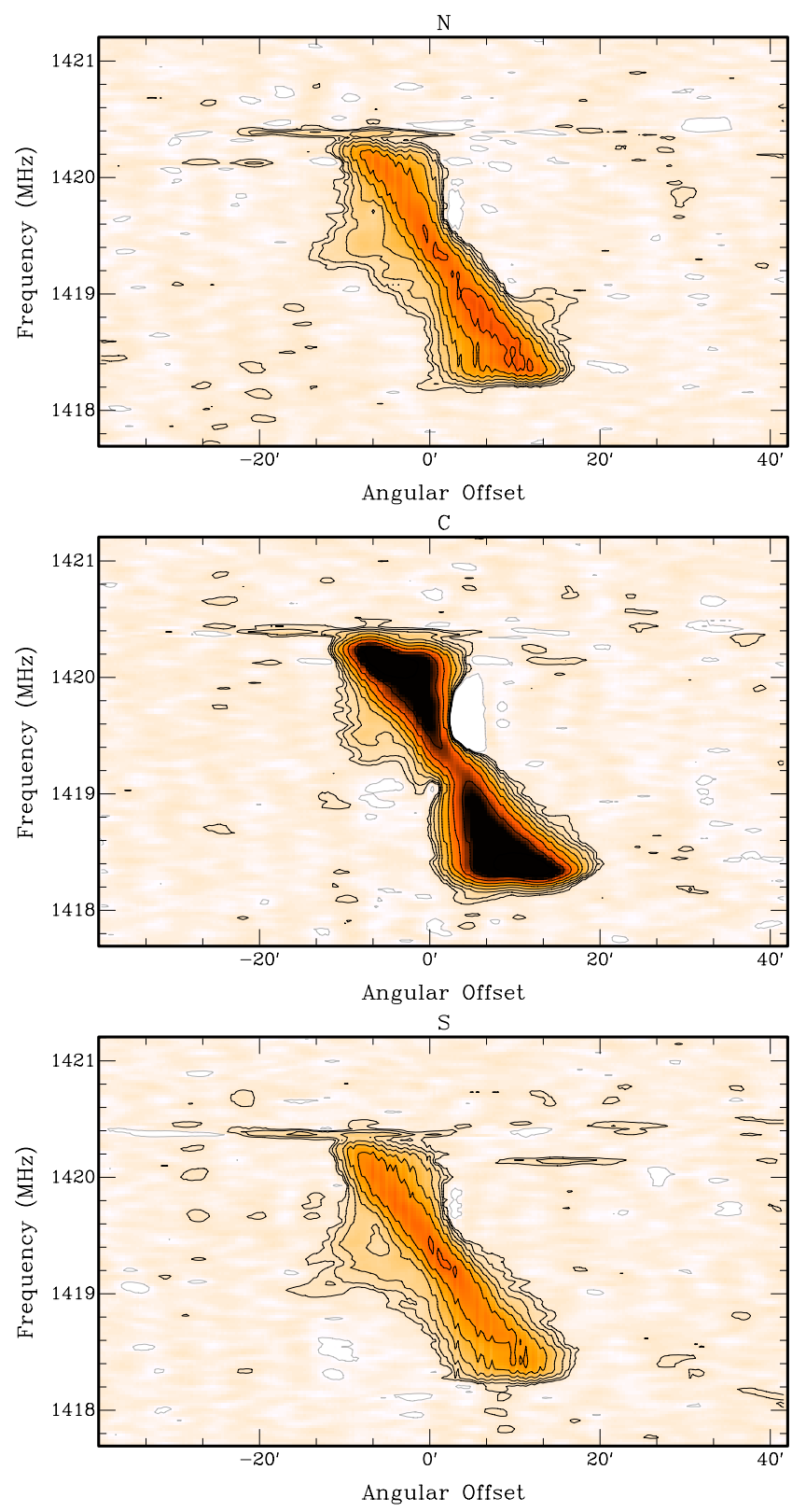

Figure 11. PV slices parallel to the major axis. One is on the centre while the other two are $4^{\prime} \mathrm{NW}$ and SE of the major axis along the minor axis. Contour levels are $-3,3,6,12,24,48,96 \mathrm{mJy} /$ beam

row. Based on these results, we adopt $i=76^{\circ}$ as the preferred inclination for the HI disk of NGC 253. This inclination, together with the rotation curve from the tilted ring model, accurately describes the kinematics of the HI disk.

\subsubsection{Comparison of the RC with the data}

Finally, the best test to check that our RC accurately represents the kinematics of the disk is to look at the superposition of the receding and the approaching sides on a Position-Velocity (PV) diagram obtained along the major axis, as shown in Figure 15 (top panel). It can be seen that the deprojected velocities follow very closely the ridge of the emission, which shows that our $\mathrm{RC}$ is a good representation of the kinematics on the major axis. Note on the PV diagram the anomalous $\mathrm{HI}$ around $200 \mathrm{~km} \mathrm{~s}^{-1}$. We should point out the presence of Galactic HI, which can be seen around $0 \mathrm{~km} \mathrm{~s}^{-1}$. Even though we did exclude the channels with obvious Galactic emission, it can be seen that some of it is still present. However, since there is much more $\mathrm{HI}$ on the receding side than on the approaching side, very few data points from the approaching side contribute to the last points of the solution for both sides (cf. Fig. 13) and make us confident in our adopted RC.

One last point needs to be emphasized. At the beginning of the section, we mentioned that a $|\cos \theta|^{2}$ weighting function and an exclusion angle of $60^{\circ}$ about the minor axis have been used to minimize the influence of large deprojection errors close to the minor axis in view of the large inclination of the galaxy. Typically, when a galaxy is not highly inclined (p.e. $i=30-45^{\circ}$ ), all the data can be used. For intermediate inclinations (p.e. $i=45-60^{\circ}$ ), a free angle of $\sim 30^{\circ}$ and $\mathrm{a}|\cos \theta|$ weighting function should be used to give 


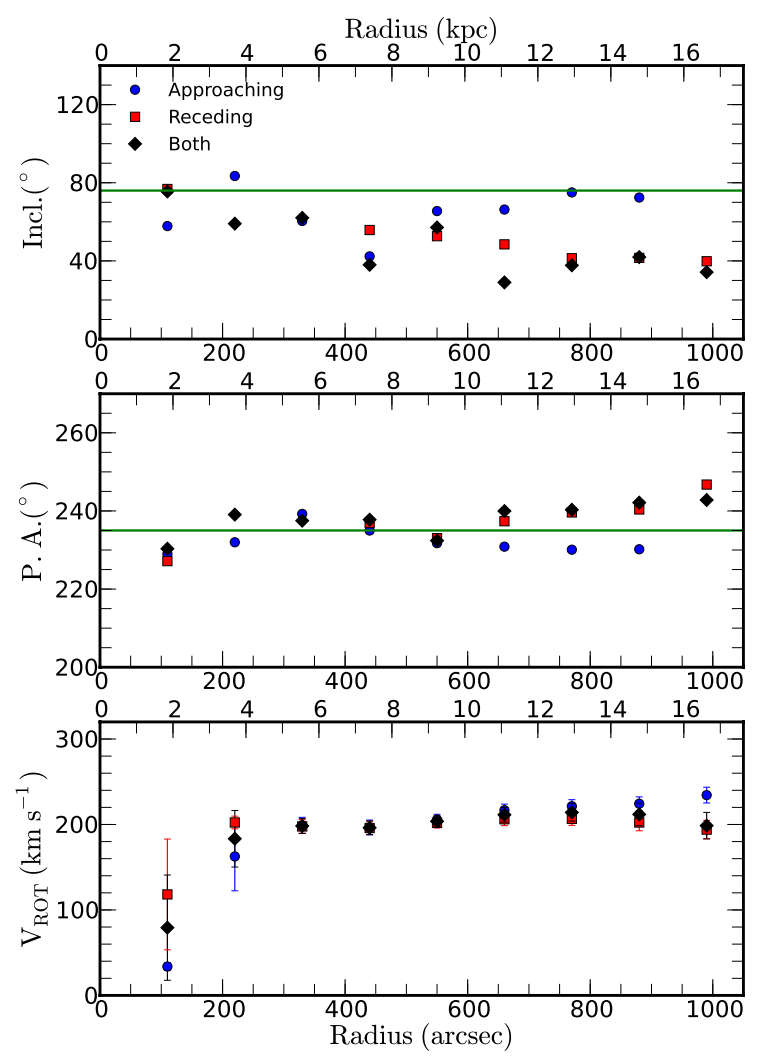

Figure 13. ROTCUR solution and derived RC for the HI disk.The green lines show our adopted $P A=235^{\circ}$ and $i=76^{\circ}$.

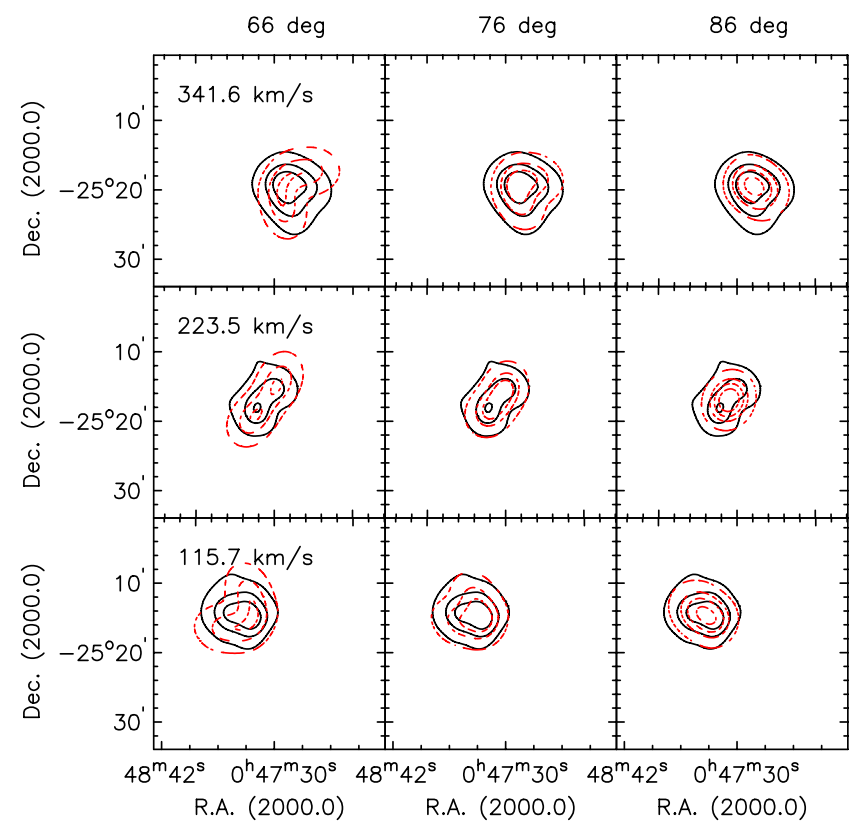

Figure 14. Comparison of the data (black) with 3D-models (red-dashed) using ROTCUR solutions with $i=66^{\circ}$ (left), $76^{\circ}$ (centre) and $86^{\circ}$ (right) for 3 different channels. The contours are at levels of $0.03,0.2,0.4,0.7$ Jy/beam.
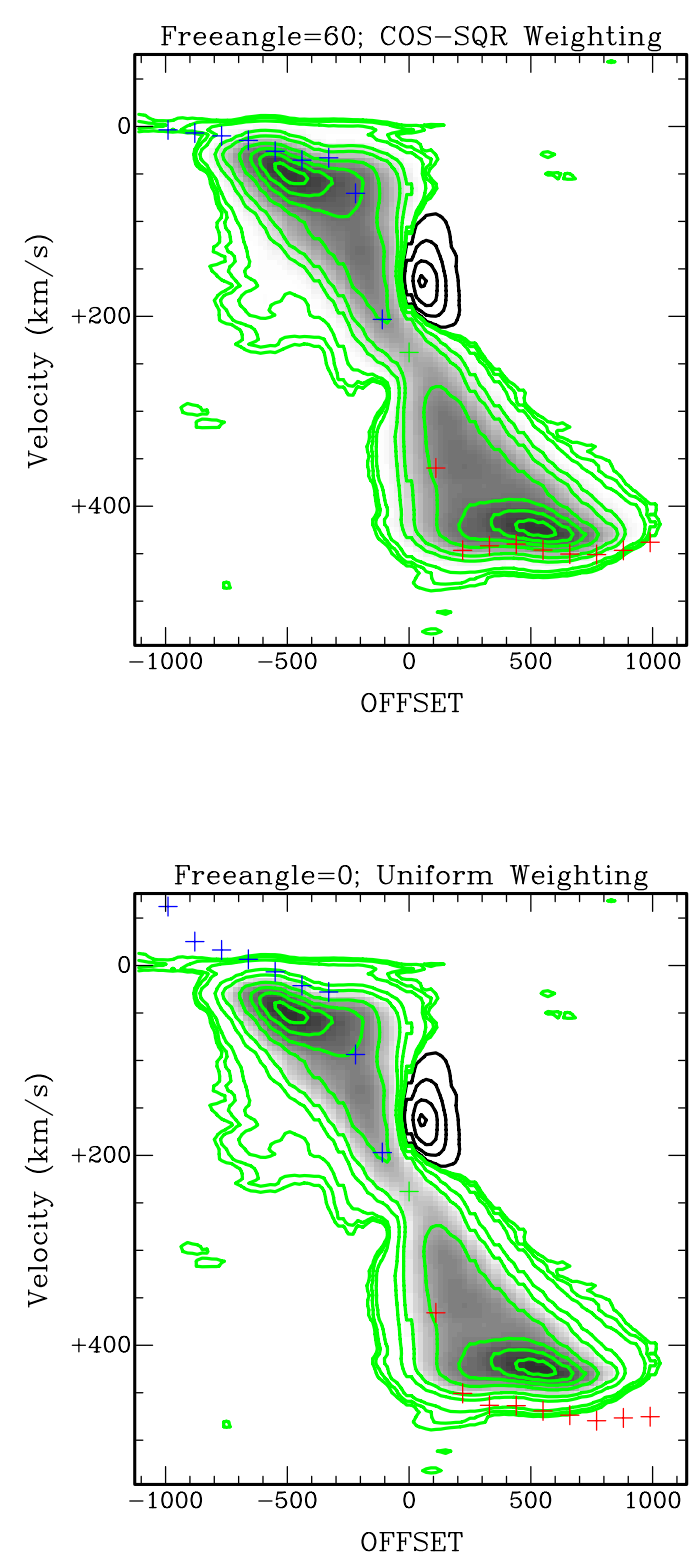

Figure 15. PV diagrams along the major axis with the receding (red pluses) and approaching (blue pluses) RC superimposed using the adopted kinematical parameters with a free angle of $60^{\circ}$ and a $|\cos \theta|^{2}$ weighing function at the top and no free angle and no weighing function at the bottom. The black contours are negative contours around the absorption feature.

more weight to the data points close to the major axis. However, for high inclinations (p.e. $i>75^{\circ}$ ), it is suggested to use a larger exclusion angle $\left(\sim 60^{\circ}\right)$ and a $|\cos \theta|^{2}$ weighting function.

To illustrate this, we ran a ROTCUR model for NGC 253 with no exclusion angle and no weighting function. The resulting RCs for the approaching and receding sides are overlaid on the PVdiagram of the major axis in the bottom panel of Figure 15. We see that this solution is inconsistent with the kinematics observed on the major axis and must be discarded. This illustrates well that one has to be very careful when deriving the kinematics of highly inclined galaxies, especially with ROTCUR. 


\begin{tabular}{ccc}
\hline \hline $\begin{array}{c}\text { Radius } \\
(\operatorname{arcsec})\end{array}$ & $\begin{array}{c}\mathrm{V}_{\text {rot }} \\
\left(\mathrm{km} \mathrm{s}^{-1}\right)\end{array}$ & $\begin{array}{c}\Delta \mathrm{V} \\
\left(\mathrm{km} \mathrm{s}^{-1}\right)\end{array}$ \\
\hline 110 & 79.3 & 61.6 \\
220 & 183.3 & 33.1 \\
330 & 198.3 & 8.6 \\
440 & 196.2 & 8.0 \\
550 & 203.7 & 6.4 \\
660 & 211.4 & 8.7 \\
770 & 214.1 & 10.1 \\
880 & 211.8 & 13.2 \\
990 & 198.6 & 15.5 \\
1100 & 186.7 & 16.5 \\
\hline
\end{tabular}

Table 7: Rotation velocities and errors for the HI disk of NGC 253.

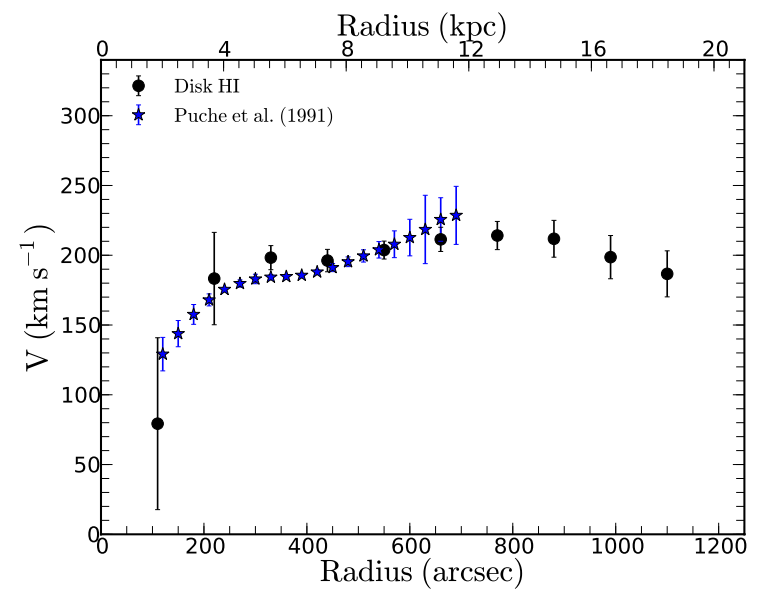

Figure 16. Comparison of the VLA \& KAT-7 rotation curves.

Figure 16 compares our adopted RC for the disk with the one derived by Puche, Carignan \& van Gorkom (1991) using VLA observations. We see that out to $\mathrm{r} \sim 12 \mathrm{kpc}$ (the last measured velocity point with the VLA data), both rotation curves agree well within the errors, with the exception of our velocity at $5.5^{\prime}$, which is $\sim 15 \mathrm{~km}$ $\mathrm{s}^{-1}$ larger. As for the KAT-7 RC, it rises to a velocity of $\sim 200 \mathrm{~km}$ $\mathrm{s}^{-1}$ at $5.5^{\prime}$, stays more or less flat out to $\sim 15^{\prime}$ and than declines by $\sim 20 \mathrm{~km} \mathrm{~s}^{-1}$ out to the last point. We will discuss this possible decline in Sec. 7.1.

\subsection{Tilted-ring model for the anomalous $\mathrm{HI}$}

The kinematical solution for the halo gas is shown in Figure 17. Tilted ring models were fitted to the velocity field of the anomalous HI (shown at the bottom of Fig. 12). When allowed to freely vary with radius, it is seen that $P A$ and $i$ are much better constrained for the anomalous component than for the disk. Despite the small change of $P A$ for the last two points, it was decided to adopt constant $P A$ and $i$ (green lines). The rotation velocities for the anomalous gas are given in Table 8 and are shown in blue in Figure 18, along with the rotation velocities for the disk. It is different from the RC seen in the bottom panel of Fig 17 because $P A$ and $i$ were kept fixed (green lines).

This $\mathrm{RC}$ for the anomalous gas is surprisingly very regular. It rises out to $\sim 15 \mathrm{kpc}$ and then declines slowly out to the last measured velocity point. In the outer parts, the anomalous gas is

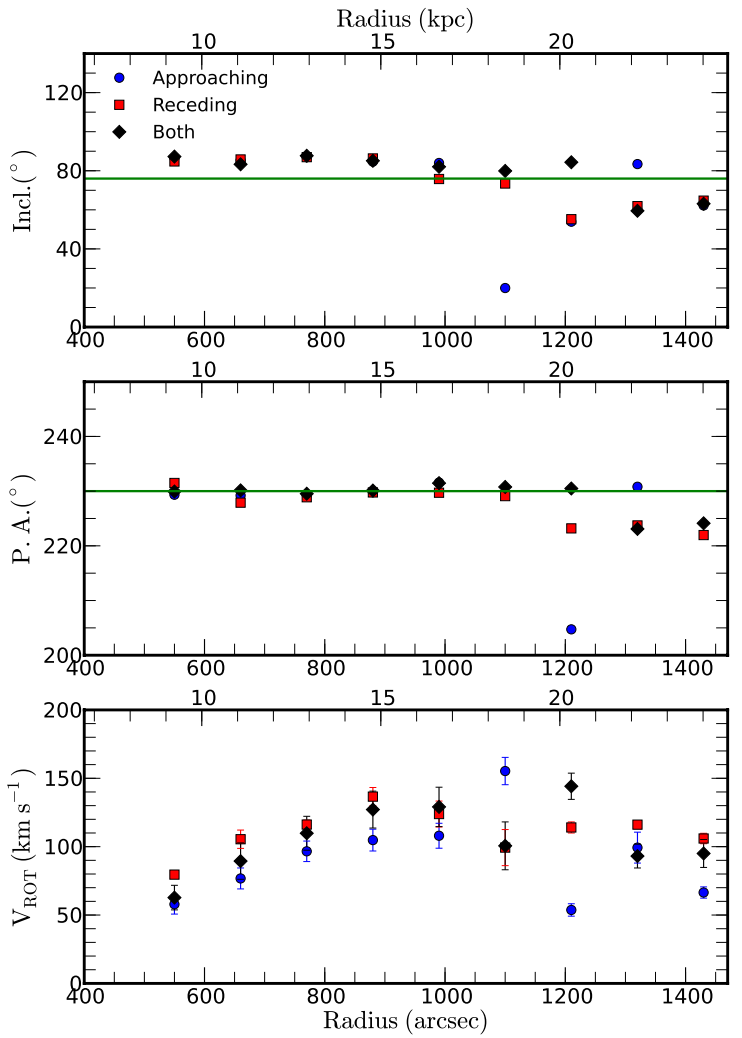

Figure 17. ROTCUR solution and derived RC for the anomalous HI. The green lines show our adopted $P A=230^{\circ}$ and $i=76^{\circ}$.

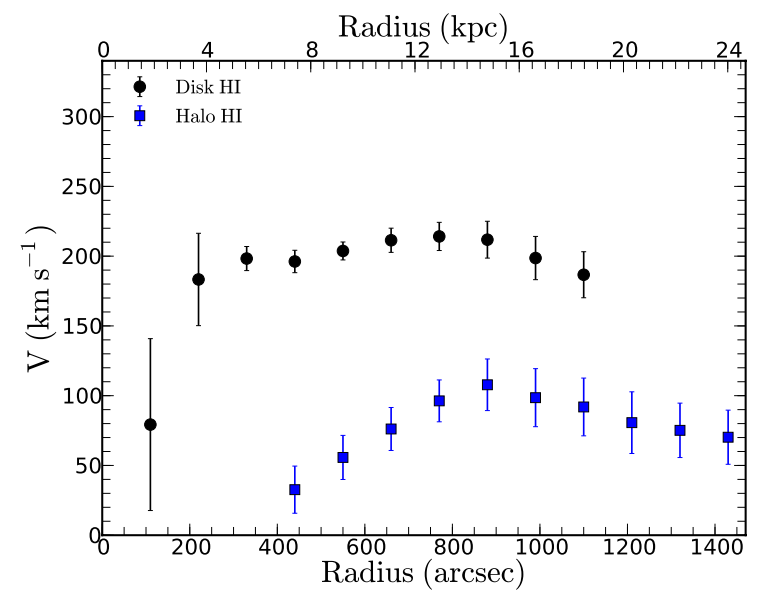

Figure 18. Comparison of the disk kinematics and of the anomalous $\mathrm{HI}$ kinematics.

kinematically lagging the disk gas by $\sim 100 \mathrm{~km} \mathrm{~s}^{-1}$. Such lagging of extra-planar gas is also seen in other galaxies (see e.g. Elson 2014; Hess et al. 2009; Heald et al. 2007; Fraternali et al. 2001). 


\begin{tabular}{ccc}
\hline \hline $\begin{array}{c}\text { Radius } \\
(\operatorname{arcsec})\end{array}$ & $\begin{array}{c}\mathrm{V}_{\text {rot }} \\
\left(\mathrm{km} \mathrm{s}^{-1}\right)\end{array}$ & $\begin{array}{c}\Delta \mathrm{V} \\
\left(\mathrm{km} \mathrm{s}^{-1}\right)\end{array}$ \\
\hline 440 & 32.6 & 16.9 \\
550 & 55.7 & 15.8 \\
660 & 76.1 & 15.4 \\
770 & 96.3 & 15.0 \\
880 & 107.8 & 18.5 \\
990 & 98.6 & 20.8 \\
1100 & 91.9 & 20.7 \\
1210 & 80.7 & 22.1 \\
1320 & 75.2 & 19.5 \\
1430 & 70.2 & 19.4 \\
\hline
\end{tabular}

Table 8: Rotation velocities and errors for the anomalous HI of NGC 253.

\section{DISCUSSION}

\subsection{Is the Rotation Curve of NGC 253 really declining ?}

Since the discovery in the 1970s that the RC of most spiral galaxies remains flat out to their last measured point (Freeman 1970; Bosma 1981), the obvious question is: where do the disks of spiral galaxies end? Especially in the case of spiral galaxies, the HI surface density declines with increasing radius to a point where it decreases abruptly and the HI disk seems to be truncated. This truncation is usually around surface densities $\sim 10^{18}-10^{19} \mathrm{~cm}^{-2}$ (HI self-shielding limit). This does not necessarily mean that there is no more hydrogen further out. As suggested by Bland-Hawthorn, Freeman \& Quinn (1997), it may just be that the ambient radiation field out there is sufficiently strong that cold gas gets ionized (Maloney 1990; Silk \& Sunyaev 1976). While the UV background radiation may not be sufficiently powerful to be responsible for this ionization, the situation may be different in a starburst object like NGC 253.

We have seen in Sec. 4 that the HI disk of NGC 253 only extends out to the optical radius and the WISE mid-IR extent. But if, like most late-type spirals, there is hydrogen further out and if, also as seen in most spirals, the cold gas disk is warped, it could be exposed to the intense UV radiation of the starburst nucleus and be ionized. This was the motivation for Bland-Hawthorn, Freeman \& Quinn (1997) and Hlavacek-Larrondo et al. (2011) to try to detect $\mathrm{H} \alpha$ emission in the outer parts of NGC 253 using Fabry-Perot (FP) interferometry, and perhaps succeed to find where the mass (luminous \& dark) ends.

Both groups detected $\mathrm{H} \alpha$ at the limit of the HI disk (at 690, 720 and 900 arcsec) but Hlavacek-Larrondo et al. (2011) also detected $[\mathrm{NII}]$ out to 1140 arcsec or $1.4 \mathrm{D}_{25}$ on the receding side of the galaxy. The most interesting result was that both sets of measurements imply that the RC is declining past the last previously measured HI velocity point (Puche, Carignan \& van Gorkom 1991). From this, Bland-Hawthorn, Freeman \& Quinn (1997) conclude that it suggests that the dark halo of NGC 253 may be truncated near the HI edge and provides further support for the link between dark matter and HI (Bosma 1978; Carignan \& Beaulieu 1989; Carignan \& Puche 1990a,b; Puche, Carignan \& Bosma 1990; Jobin \& Carignan 1990; Hoekstra, van Albada \& Sancisi 2001; Swaters et al. 2012; Meurer, Zheng \& de Blok 2013). Most importantly, it would provide a means to probe the gravitational potential beyond the edge of the Hi disk.

Figure 19 compares our HI RC to the optical FP velocity mea-

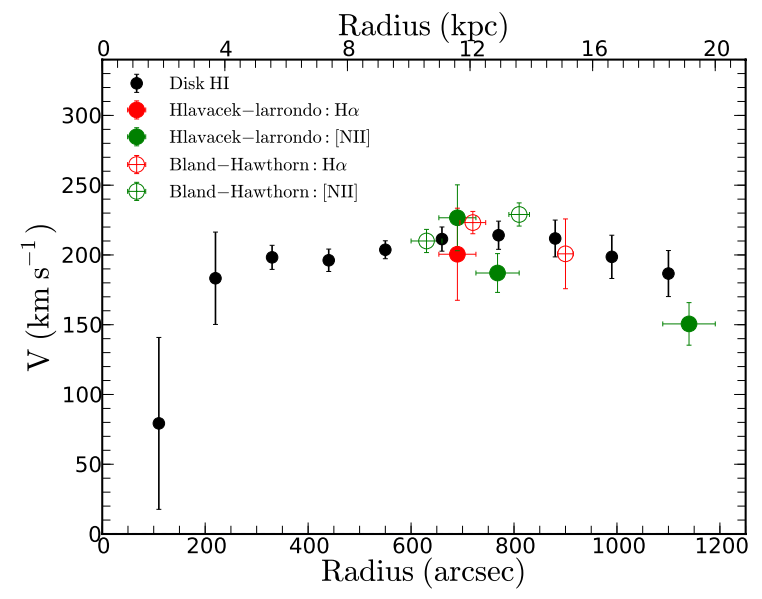

Figure 19. Comparison of the HI disk kinematics with the Fabry-Perot data of Bland-Hawthorn, Freeman \& Quinn (1997) and Hlavacek-Larrondo et al. (2011).

surements on the receding side of NGC 253. Within the errors the HI and optical velocities agree very well and seem to confirm that the RC of NGC 253 declines for $\mathrm{R} \geq 12 \mathrm{kpc}$. Could a bad choice of orientation parameters, mainly $P A$ and/or $i$, mimic a declining RC? As seen in Fig. 13 (see also Koribalski, Whiteoak \& Houghton 1995), there is a suggestion that $P A$ might be increasing for the last two rings. Tilted-ring models, run with those higher values, did not change significantly the derived velocities. However, $i$ could have a more important effect but to bring the last point at the level of the flat part of the RC, an $i \sim 56^{\circ}$ would be needed which, as shown in Sec. 6.1.1, is excluded.

NGC 253 is the second galaxy in the Sculptor group with a declining RC. Carignan \& Puche (1990a) and de Blok et al (2008) showed NGC7793 to also have a declining RC, which is contrary to what is observed for the vast majority of spiral galaxies.

\subsection{Star formation in NGC 253 using WISE data}

If we want to understand the gas flows in the starburst galaxy NGC 253 , it is important to understand its star formation. WISE imaging provides the ideal platform for global property studies of large galaxies, such as NGC 253, due to the large field of view and the broad photometric bands that cover both the stellar $(3.4$ and $4.6 \mu \mathrm{m}$, or the W1 and W2 bands) and ISM components (12 and $22 \mu \mathrm{m}$, or W3 and W4 bands). Reconstructions using a "drizzle" sampling technique (Jarrett et al. 2012) that conserves the native angular resolution ( 6 arcsec in the $3.4 \mu \mathrm{m}$ band) were carried out for NGC 253.

The resulting mosaics fully cover the galaxy and its immediate environment. However, a significant impediment to the 12 and $22 \mu \mathrm{m}$ imaging arises from the nuclear starburst itself, saturating the inner few pixels that comprise the unresolved nucleus. To recover this lost data, we apply a PSF-technique that was developed by T. Jarrett for saturation in Spitzer IRAC and MIPS imaging (see IRAC Instrument Handbook) and successfully deployed for study of the Circinus Galaxy (For, Koribalski \& Jarrett 2012). The rectified WISE imaging of NGC 253 is shown in Figure 5 and Figure 10, where all bands are combined into a four-color graphic: W1 assigned shades of blue, W2 green, W3 orange and W4 red.

Deploying the characterization pipeline of the WISE Enhanced Resolution Atlas (Jarrett et al. 2013), foreground stars are 
identified and removed, the two-dimensional shape of NGC 253 is determined and photometry is extracted. The one- $\sigma$ isophotal (23.24 Vega mag $\operatorname{arcsec}^{-2}$ or $26.6 \mathrm{mag} \operatorname{arcsec}^{-2}$ in $\mathrm{AB}$ ) diameter is found to be 42.2 arcmin with an axis ratio of 0.276 . The corresponding integrated flux densities are $12.74,8.44,47.95$, and 118.04 Jy, for W1, W2, W3 and W4 respectively, indicative of very strong PAH emission (W3) and warm dust continuum (W4) arising from the active star formation. We note that the "total" flux, as inferred from the isophotal plus extrapolated disk emission, is only a few per cent larger, consistent with a relatively truncated stellar disk.

As demonstrated in Jarrett et al. (2013) and Cluver et al. (2014), the stellar mass-to-light ratio has a simple dependence on the WISE W1 and W2 integrated fluxes. With our adopted distance of $3.47 \mathrm{Mpc}$, the corresponding W1 absolute magnitude and Log [in-band luminosity $L_{w 1}\left(L_{\odot}\right)$ ] is -24.24 and 10.99 , respectively. The W1-W2 color is $0.21 \mathrm{mag}$, and employing the M/L relation from Cluver et al. (2014), the inferred $\log$ [stellar mass $\left.\left(M_{\odot}\right)\right]$ is then 10.33, indicative of the significant (mass) evolved stellar population that represents the "back bone" of NGC 253.

Star formation activity may be gauged by the WISE 12 and $22 \mu \mathrm{m}$ luminosities, $\log \left[\mathrm{L}\left(L_{\odot}\right)\right]=9.67$ and 9.77, respectively. Here we apply the relations in Cluver et al. (2014) to estimate the dust-obscured star formation rate (SFR): 4.9 and 5.1 $M_{\odot} \mathrm{yr}^{-1}$, respectively based on the $11.3 \mu \mathrm{m}$ PAH emission and the $22 \mu \mathrm{m}$ dust continuum. Although this global SFR is typically small compared to luminous infrared galaxies, it is significant in the Local Volume, similar to the starburst M82 and to the barred grand-design spiral M83 (Jarrett et al. 2013).

We infer a star formation density of $1.7 \mathrm{M}_{d o t} / \mathrm{year} / \mathrm{kpc}^{2}$ for the central kpc of NGC 253, consistent with a nuclear starburst. For normal galaxies, Cluver et al. (2010) showed a clear linear trend in global SFR relative to the HI gas content, effectively the KennicuttSchmidt law. Starburst galaxies, such as M82 and NGC 253, deviate from this relation due to the enhanced nuclear starburst phase, as seen in Figure 20. Finally, normalizing the SFR with the stellar mass, the resulting $\log$ [specific SFR $\left(\mathrm{yr}^{-1}\right)$ ] is -9.86 and -9.83 , respectively $\mathrm{W} 3$ and $\mathrm{W} 4$. This disk building rate is relatively high for nearby galaxies - NGC 253 is still actively building its disk and bulge $\mathrm{D}$ and is comparable to other large star forming spirals, notably M83 and NGC 6946 (Jarrett et al. 2013).

\subsection{Origin of the Anomalous $\mathrm{HI}$ : inflow or outflow?}

As discussed in the introduction, at least three possible scenarios could be envisaged to explain the anomalous (halo) gas that is observed. The first one is the classical galactic fountain outflow scenario, where gas is being expelled from the disk through multiple supernova explosions from clusters of massive stars (Schapiro \& Field 1976). The second one is the inflow scenario of gas of external origin in the form of primordial gas clouds left over from the formation of the galaxy (Oort 1966). Finally, that halo gas could come from ISM torn out of dwarf galaxies during a close encounter with NGC 253 (Putman, Peek \& Joung 2012).

It is clear from this study that the first scenario can explain a large part of the anomalous gas observed. The known starburst nature of NGC 253, with its SFR $\geq 5 \mathrm{M}_{\odot} \mathrm{yr}^{-1}$ (see Sec.7.2) and the large amount of hot halo gas seen along the minor axis in the X-ray observations (Pietsch et al. 2000), all point to gas being expelled from the nuclear region. This starburst-driven super wind was studied in great detail by Westmoquette, Smith \& Gallagher (2011) through deep $\mathrm{H} \alpha$ imaging with the MPG/ESO $2.2 \mathrm{~m}$ WFI

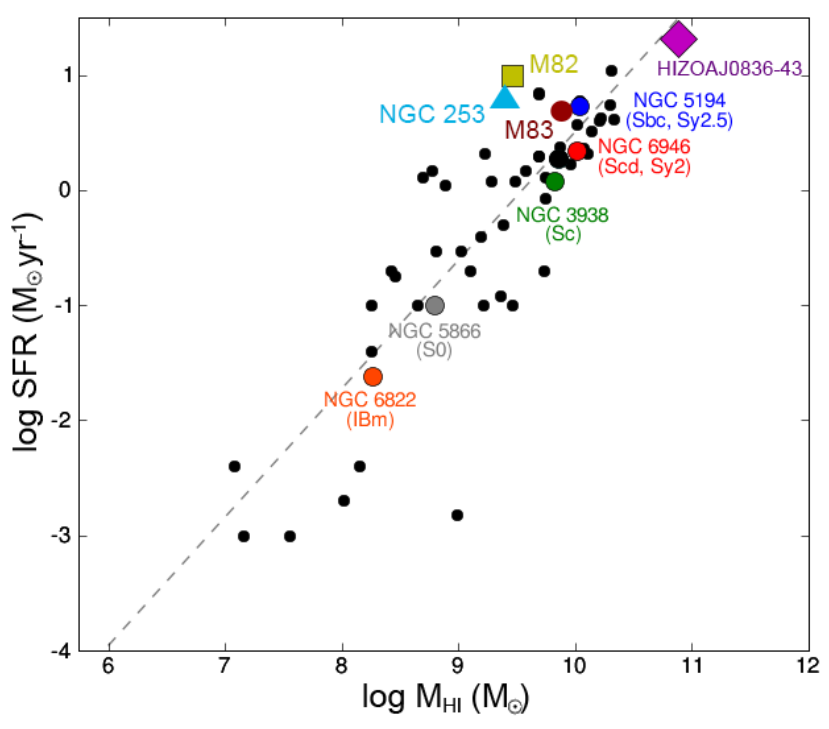

Figure 20. Obscured star formation rate compared to the total neutral hydrogen content. Adapted from Cluver et al. (2010), the sample is from SINGS (Kennicutt et al 2003) with highlighted galaxies, notably nearby starbursts M82 and NGC 253 (this work).

and optical spectroscopy with the VLT/VIMOS-IFU and with the WIYN/SparsePak IFU. They investigate the known minor axis outflow cone, which is well-defined in the $\mathrm{H} \alpha$ imaging and kinematics between radii of 280 and 660 pc from the nucleus. Kinematic modelling indicates a wide opening angle $\left(\sim 60^{\circ}\right)$, an inclination consistent with that of the disc and deprojected outflow speeds of a few $100 \mathrm{~km} \mathrm{~s}^{-1}$ that increase with distance above the plane.

A dual origin for the gas in the halo in the form of galactic fountains originating within the boiling star-forming disk and a strong galactic super wind emanating from the nuclear starburst, has previously been suggested by Pietsch et al. (2000). It is thus quite possible that the classical galactic fountain (ionized outflow seen in the $\mathrm{H} \alpha$ emission, cooling down through radiative processes and raining back onto the disk) provides most of the extra planar gas up to 5-10 kpc, while the gas higher up in the halo could originates from the starburst region, expelled as hot gas and then cooled to its neutral form. The most convincing piece of evidence that supports the outflow origin for the extra planar gas is seen in Figure 12 , where it is apparent that the halo gas retained the kinematical signature of the disk.

As suggested by a referee, the location of the anomalous gas, situated more toward the edge of the disk (see Fig. 10) could possibly be interpreted as the result of the superwind outflow, as in the case of NGC 1482 (Hota \& Saikia 2005). It looks as if the anomalous gas in the halo is pushed by this outflow and is providing collimation to the outflowing gas. This halo gas would have a different origin than the outflow gas, most probably through galactic fountains, which is suggested by the observation that it retains traces of the main disk kinematics.

If the anomalous gas was an inflow from external origin, it is expected that those clouds would include material at forbidden velocities. After a careful visual search of the HI cube of NGC 253, no such clouds with that expected peculiar kinematics were uncovered. However, looking at the size of the inflow clouds, e.g. in NGC 891 (Oosterloo, Fraternali \& Sancisi 2007), of only 1-2', such clouds would most probably not be detected in our data cube because of beam dilution (beam $\simeq 3.5^{\prime} \times 3^{\prime}$ ). Thus, we cannot exclude com- 
pletely the presence of such small inflow clouds. Finally, our data seems also to exclude gas torn off a dwarf galaxy during a close encounter since again we would not necessarily expect that gas to have the same kinematics as that of the disk.

The last question left to answer: where spatially is most of the detected anomalous gas? Looking at Fig. 18, it can be seen that the halo gas has a much shallower gradient than the disk gas and is lagging by $\sim 100 \mathrm{~km} \mathrm{~s}^{-1}$ in the outer parts. Modelling done (see e.g. Fraternali et al. 2005) of extra planar gas predicts a lag of $\sim 15$ $\mathrm{km} \mathrm{s}^{-1} \mathrm{kpc}^{-1}$, consistent with observations of edge-on galaxies. Applied to NGC 253, this suggests that the bulk of the gas should be around $6-8 \mathrm{kpc}$ from the plane of the main $\mathrm{HI}$ disk. If it were closer to the disk (see e.g. Hess et al. 2009), it would have a steeper gradient in the inner parts and a much smaller lagging velocity.

\subsection{Origin of the starburst nature of NGC 253}

One of the principal reasons often given for high SFR is galaxygalaxy interaction (see e.g. Larson \& Tinsley 1978), whether in the form of merger, close encounter or interaction with the IGM. A good example is the M81 group where the three core members M81, M82 and NGC 3077 are known to be closely interacting from HI studies (van der Hulst 1979; Yun, Ho \& Lo 1994) and, at the same time, all three show evidence for an AGN or starburst activity, most likely induced by the on-going tidal interactions.

In contrast, the environment of NGC 253 appears much more devoid of any sign of gravitational interaction either with low-mass companions or the IGM. The search of Côté et al. (1991) for dwarf galaxies in the Sculptor group (see their Fig. 4) do not show any obvious nearby dIrr with HI emission, while a few possible dSphs candidates were identified. As for the presence of a significant IGM component, the study by Putman et al. (2003) suggests that the surrounding region has very little intergalactic gas (see their Fig. 12).

However, the apparent lack of evidence of any actual interaction does not tell us anything about possible past interactions of NGC 253 with its environment. For example, as shown by Whiting (1999), the spin of the NGC 253 disk is consistent with torquing from NGC 247 , which is its closest ( $~ 350 \mathrm{kpc}$ ) large companion. Moreover, both galaxies appear to have truncated HI disks (Puche, Carignan \& van Gorkom 1991; Carignan \& Puche 1990b), since they barely extend out to their optical diameter, This is contrary to what is generally seen in late-type spirals where the HI disk is usually much more extended than their stellar disk (Sancisi 1983). As an example, the HI disk of NGC 300, another late-type spiral in the Sculptor group, has a diameter more than 1.5 times the optical diameter (Puche, Carignan \& Bosma 1990).

Despite its apparent isolation, there is some evidence which suggests that NGC 253 may have been recently involved in a merger. For example, in a recent X-ray study to spatially resolve the starburst region of NGC 253 (Wik et al. 2014), the 4-6 keV data clearly shows what appears to be a double nucleus (see their Fig. 4). Earlier studies (Anantharamaiah \& Goss 1996; Prada, Gutierrez \& McKeith 1998) also found evidence of two dynamically distinct systems close to the centre of NGC 253. As discussed by Davidge (2010), one system has an axis of rotation that differs from that of the galaxy disk, while the other appears to be counter-rotating with respect to the galaxy disk. These are all signatures of a recent merger. Das, Anantharamaiah \& Yun (2001) estimate that the merger may have involved the accretion of $10^{6} \mathrm{M}_{\odot}$ of material $\sim 10^{7}$ years ago. In view of the actual isolation of NGC 253 , this is the most likely explanation for the starburst activity in its nucleus.

\section{SUMMARY AND CONCLUSIONS}

This study presents the results of $\sim 150$ hours of KAT- 7 observations in HI line mode, of which $\sim 115$ hours were spent on NGC 253 . For the analysis, the data were smoothed to a velocity resolution of $5 \mathrm{~km} \mathrm{~s}^{-1}$ and the spatial resolution (synthesized beam) is $213^{\prime \prime} \times 188^{\prime \prime}$. As much as $33 \%$ more flux was detected by KAT7, compared to previous VLA observations for a total HI mass of $2.1 \pm 0.1 \times 10^{9} \mathrm{M}_{\odot}$. A sensitivity limit of $\sim 1.0 \times 10^{19} \mathrm{~cm}^{-2}$ in column density allows us to uncover a large quantity of extra planar gas out to projected distances from the plane of $\sim 9-10 \mathrm{kpc}$ in the centre and 13-14 kpc at the edge of the disk. However, in the radial direction, the HI disk is relatively small for a late-type spiral, similar to the optical and mid-IR disk, consistent with the truncation scenario.

A robust method was developed to separate the regular and anomalous HI components in NGC 253. This method, which used interactive profile fitting in combination with PV diagrams, allowed to fit separately the different components pixel by pixel. For the disk, the RC derived from the KAT-7 data agrees well with the VLA data out to $\sim 12 \mathrm{kpc}$ (the last measured VLA velocity point). For $\mathrm{R}$ $>12 \mathrm{kpc}$, the RC declines by $\sim 20 \mathrm{~km} \mathrm{~s}^{-1}$ out to the last point at $\sim 18 \mathrm{kpc}$. This decline, observed in the outer parts, agrees with previous optical FP observations. As for the halo gas, which extends out to $\sim 24 \mathrm{kpc}$, its $\mathrm{RC}$ has a very shallow gradient in the inner parts and is kinematically lagging the disk gas by $\sim 100 \mathrm{~km} \mathrm{~s}^{-1}$ in the outer parts. That extra planar cold gas component is seen at the edge of the hot gas component revealed by the X-ray observations.

Our results point towards an outflow scenario for the origin of the extra planar gas. This outflow has two origins. First, as clearly seen in X-ray observations, a lot of hot gas is expelled in the halo from the nuclear starburst region. As the gas expands, it eventually converts back to $\mathrm{HI}$ as it cools through radiative losses; raining back down on the disk and feeding subsequent star formation. Secondly, another part of that extra planar gas has most likely a galactic fountain origin from the star-forming disk.

The KAT-7 observations presented in this work clearly show that despite its relatively small size ( 7 x $12 \mathrm{~m}$ antennae), this telescope occupies a niche for detecting large scale low surface brightness diffuse emission over the $\sim 1^{\circ}$ FWHM of its antennae. It should be kept in mind that this telescope was built primarily as a testbed for MeerKAT and the SKA such that any scientific result that can be obtained is a bonus. While most of the extragalactic $\mathrm{HI}$ sources would be unresolved by the $\sim 4^{\prime}$ synthesized beam, many projects such as this one and the project on NGC 3109 (Carignan et al. 2013) can be done on nearby very extended objects such as Local Group galaxies or galaxies in nearby groups like Sculptor.

\section{ACKNOWLEDGMENTS}

We thank the entire SKA SA team for allowing us to obtain scientific data during the commissioning phase of KAT-7. The work of $\mathrm{CC}$ an $\mathrm{TJ}$ is based upon research supported by the South African Research Chairs Initiative (SARChI) of the Department of Science and Technology (DST), the Square Kilometre Array South Africa (SKA SA) and the National Research Foundation (NRF). The research of DL, EE \& TR have been supported by SARChI, SKA SA fellowships. 


\section{REFERENCES}

Allen, R.J., Baldwin, J.E., Sancisi, R., 1978, A\&A, 62, 397

Anantharamaiah, K.R., Goss, W.M., 1996, ApJ, 466, L13

Barbieri, C.V., Fraternali, F., Oosterloo, T., Bertin, G., Boomsma,

R., Sanciisi, R., 2005, A\&A, 439, 947

de la Beaujardière, O., Kazès, I., Le Squeren, A.M., Nguyen-

Quang-Rieu, 1968, Annales d'Atrophysique, 31, 387

Begeman, K.G., 1987, PhD thesis, Rijsuniversiteit Groningen

Begeman, K.G., 1989, A\&A, 223, 47

Bland-Hawthorn, J., Freeman, K.C., Quinn, P. J., 1997, ApJ, 490, 143

Bolatto, A.D. et al., 2013, Nature, 499, 450

Boomsma, R., Oosterloo, T.A., Fraternali, F., van der Hulst, J.M.,

Sancisi, R., 2005, A\&A, 431, 65

Boomsma, R. et al., 2008, A\&A, 490, 555

Bosma, A., 1978, PhD thesis, Rijksuniversiteit Groningen

Bosma, A., 1981, AJ, 86, 1825

Bregman, J.N., 1980, ApJ, 236, 577

Carilli, C.L., Holdaway, M.A., Ho, P.T.P., De Pree, C.G., 1992, ApJ, 399, L59

Carignan, C., Beaulieu, S., 1989, ApJ, 347, 760

Carignan, C., Puche, D., 1990a, AJ, 100, 394

Carignan, C., Puche, D., 1990b, AJ, 100, 641

Carignan, C., Frank, B.S., Hess, K.M., Lucero, D.M., Randriamampandry, T.H., Goedhart, S., Passmoor, S.S., 2013, AJ, 146, 48

Cecil, G., Bland-Hawthorn, J., Veilleux, S. \& Filippenko, A. V. 2001, ApJ, 555, 338

Cluver, M.E., Jarrett, T.H., Kraan-Korteweg, R.C., Koribalski, B.S., Appleton, P.N., Melbourne, J., Emonts, B., Woudt, P. A., 2010, ApJ, 725, 1550

Cluver, M.E., et al., 2014, ApJ, 782, 90

Combes, F., Gottesman, S.T., Weliachew, L., 1977, A\&A, 59, 181

Condon, J.J., 1987, ApJSS, 65, 485

Côté, S., Freeman, K.C., Carignan, C., Quinn, P. J., 1991, AJ, 114, 1313

Dalcanton, J.J. et al., 2009, ApJS, 183, 67

Das, M., Anantharamaiah, K.R., Yun, M. S., 2001, ApJ, 549, 896

Davidge, T.J., 2010, ApJ, 725, 1342

de Blok, W.J.G., Walter, F., Brinks, E., Trachternach, C., Oh, S.H., Kennicutt, JR, R.C., 2008, AJ, 136, 2648

de Vaucouleurs, G., de Vaucouleurs, A., Corwin, H.G., Jr, Buta, R.J., Paturel, G., Fouqué, P., 1991, Third Reference Catalogue of Bright Galaxies, Vols. 1-3 (Berlin: Springer)

Ekers, R.D., Sancisi, R., 1977, A\&A, 54, 973

Elson, E.C., 2014, MNRAS, 437, 3736

Engelbracht, C.W., Rieke, M.J., Rieke, G.H., Kelly, D.M., Achtermann, J. M., 1998, ApJ, 505, 639

For, B.-Q., Koribalski, B.S., Jarrett, T. H., 2012, MNRAS, 425, 1934

Fraternali, F., Oosterloo, T., Sancisi, R., Swaters, R., 2005, in Braun R., ends, ASP Conf. Ser. Vol. 331, Extra-Planar Gas, Astron. Soc. Pac., San Francisco, p. 239

Fraternali, F., Oosterloo, T., Sancisi, R., van Moorsel, G., 2001, ApJ, 562, L47

Freeman, K.C., 1970, ApJ, 160, 811

Gentile, G. et al., 2013, A\&A, 554, A125

Greisen, E.W., 2003, in Heck, A., eds, Astrophysics and Space Science Library Vol. 285, Information Handling in Astronomy, Kluwer Academic Publishers, Dordrecht, p. 109

Heald, G., Rand, R.J., Benjamin, R.A., Bershady, A., 2007, ApJ,
663,933

Heald, G. et al., 2011, A\&A, 526, A118

Heald, G., 2012, ApJ, 754, L35

Heald, G. and the HALOGAS Team, 2014, preprint (arXiv:1409.7599v1)

Heckman, T.M., Armus, L., Miley, G. K., 1990, ApJS, 74, 833

Heesen, V., Beck, R., Krause, M., Dettmar, R.-J., 2011, A\&A, 535, A79

Heidmann, J., 1963, Annales d'Atrophysique, 26, 343

Hess, K.M., Pisano, D.J., Wilcots, E.M., Chengular, J.N., 2009, ApJ, 699, 76

Hlavacek-Larrondo, J., Carignan, C., Daigle, O., de DenusBaillargeon, M.-M., Marcelin, M., Epinat, B., Hernandez, O., 2011, MNRAS, 411, 71

Hislop, L., et al., 2011, ApJ, 733, 75

Hoekstra, H., van Albada, T.S., Sancisi, R., 2001, MNRAS, 323, 453

Hota, A., Saikia, D.J. 2005, MNRAS, 356, 998

Houghton, S., Whiteoak, J.B., Koribalski, B., Booth, R., Wiklind, T., Wielebinski, R., 1997, A\&A, 325, 923

Huchtmeier, W.K., 1972, A\&A, 17, 207

Huchtmeier, W.K., 1975, A\&A, 45, 259

Hummel, E., Smith, P., van der Hulst, J. M., 1984, A\&A, 137, 138

Hummel, E., Sancisi, R., Ekers, R. D., 1984, A\&A, 133, 1

Jacobs, B. A., et al., 2009, AJ, 138, 332

Jarrett, T. H., Chester, T., Cutri, R., Schneider, S.E., Huchra, J.P., 2003, AJ, 125, 525

Jarrett, T. H., et al., 2012, AJ, 144, 68

Jarrett, T. H., et al., 2013, AJ, 145, 6

Jobin, M., Carignan, C., 1990, AJ, 100, 648

Kamphuis, J., Briggs, F., 1992, A\&A, 253, 335

Kennicutt, R.C., Jr. et al., 2003, PASP, 115, 928

Koribalski, B., Whiteoak, J.B., Houghton, S., 1995, Proceedings

of the Astronomical Society of Australia, 12, 20

Koribalski, B.S., et al., 2004, AJ, 128, 16

Larson, R.B., Tinsley, B.M., 1978, ApJ, 219, 46

Lee, S.-W., Irwin, J.A., Dettmar, R.-J., Cunningham, C.T., Golla, G., Wang, Q.D., 2001, A\&A, 377, 759

Maloney, P., 1990, In NASA Ames Research Center, The Interstellar Medium in External Galaxies: Summaries of Contributed Papers, p 1-3

Malin, D., Hadley, B., 1997, Proceedings of the Astronomical Society of Australia, 14, 52

Marasco, A., Fraternali, F., Binney, J.J., 2012, MNRAS, 419, 1107

Mathewson, D.S., Cleary, M.N., Murray, J. D., 1974, ApJ, 190, 291

McMullin, J.P., Waters, B., Schiebel, D., Young, W., Golap, K. 2007, in Shaw, R.A., Hill F., Bell D.J., eds, ASP Conference Series Vol. 376, Astronomical Data Analysis Software and Systems XVI, Astron. Soc. Pac., San Francisco, p. 127

Meurer, G.R., Zheng, Z., de Blok, W.J.G., 2013, MNRAS, 429, 2537

Mitsuishi, I., Yamasaki, N.Y., Takei, Y., 2013, PASJ, 65, 44

Mouchine, M., Ferguson, H.C., Rich, R.M., Brown, T.M., Smith, T. E., 2005, ApJ, 633, 810

Normandeau, M., Taylor, A.R., Dewdney, P. E., 1996, Nature, 380,687

Oort, J. H., 1966, Bulletin of the Astronomical Institutes of the Netherlands, 18, 421

Oosterloo, T., Fraternali, F., Sancisi, R., 2007, AJ, 134, 1019

Pietsch, W., Vogler, A., Klein, U., Zinnecker, H., 2000, A\&A, 360, 24 
Prada, F., Gutierrez, C.M., McKeith, C. D., 1998, ApJ, 495, 765

Puche, D., Carignan, C., van Gorkom, J. H., 1991, AJ, 101, 456

Puche, D., Carignan, C., Bosma, A., 1990, AJ, 100, 1468

Puche, D., Carignan, C., 1988, AJ, 95, 1025

Putman, M.E. et al.,1998, Nature, 394, 752

Putman, M.E., Staveley-Smith, L., Freeman, K.C., Gibson, B.K.,

Barnes, D. G., 2003, ApJ, 586, 170

Putman, M.E., Peek, J.E.G., Joung, M. R., 2012, ARA\&A, 50, 491

Rekola, R. et al., 2005, MNRAS, 361, 330

Rieke, G.H., Lebofsky, M.J., Thompson, R.I., Low, F.J., Tokunaga, A. T., 1980, ApJ, 238, 24

Rieke, G.H, Lebofsky, M.J., Walker, C.E., 1988, ApJ, 325, 679

Rogstad, D.H., Lockhart, I.A., Wright, M.C.H., 1974, ApJ, 193, 309

Sakamoto, K., Mao, R.-Q., Matsushita, S., Peck, A.B., Sawada, T., Wiedner, M.C., 2011, ApJ, 735, 19

Sancisi, R., 1983, in Proc. of the IAU Symposium No. 100, Internal kinematics and dynamics of galaxies, D. Reidel Publishing Co., Dordrecht, p. 55

Sancisi, R., Fraternali, F., Oosterloo, T., van der Hulst, T., 2008, A\&AR, 15, 189

Sault, R.J., Teuben, P.J., Wright, M.C.H., 1995, in Shaw, R.A., Payne, H.E., Hayes, J.J.E., eds, ASP Conference Ser. Vol. 77, Astronomical Data Analysis Software and Systems IV, Astron. Soc. Pac., San Francisco, p. 433

Schaap, W.E., Sancisi, R., Swaters, R. A., 2000, A\&A, 356, L52

Shapiro, P.R., Field, G. B., 1976, ApJ, 205, 762

Seaquist, E.R., Odegard, N., 1991, ApJ, 369, 320

Silk, J., Sunyaev, R. A., 1976, Nature, 260, 508

Sofue, Y., Tutui, Y., Honma, A., Tomita, A., Takamiya, T., Koda, J., Takeda, Y., 1999, ApJ, 523, 136

Solanes, J.M., Giovanelli, R., Haynes, M.P. 1996, ApJ, 461, 60

Strickland, D.K., Heckman, T.M., Weaver, K.A., Hoopes, C.G., Dahlen, M., 2002, ApJ, 58, 689

Swaters, R.A., Sancisi, R., van der Hulst, J.M., 1997, ApJ, 491 140

Swaters, R.A., Sancisi, R, van der Hulst, J.M., van Albada, T. S., 2012, MNRAS, 425, 2299

Thilker, D.A. et al., 2004, ApJ, 601, L39

Ulvestad, J.S., Antonucci, R.R.J., 1997, ApJ, 488, 621

van der Hulst, J.M., 1979, A\&A, 75, 97

van der Hulst, J.M., Terlouw, J.P., Begeman, K., Zwitser, W., Roelfsema, P.R., 1992, in Worrall, D.M., Biemesderfer, C., Barnes, J., eds, ASP Conf. Ser. Vol. 25, Astron. Soc. Pac., San Francisco, p. 131

Wakker, B.P., van Woerden, H., 1997, ARA\&A, 35, 217

Westmeier, T., Braun, R., Thilker, D., 2005, A\&A, 436, 101

Westmoquette, M.S., Smith, L.J., Gallagher, J. S. III 2011, MNRAS, 414, 3719

Whiting, A.B., 1999, AJ, 117, 202

Wik, D.R. et al., 2014, preprint(asXiv:1141.1089v1)

Yun, M.S., Ho, P.T.P., Lo, K.Y., 1994, Nature, 372, 530

Zschaechner, L.K., Rand, R.J., Heald, G.H., Gentile, G., Kamphuis P., 2011, ApJ, 740, 35

Zschaechner, L.K., Rand, R.J., Heald, G.H., Gentile, G., Gyula, J. 2012, ApJ, 760, 37 